\title{
Observer-Based Bounded Control for Discrete Time-Delay Uncertain Nonlinear Systems
}

\author{
Bei Wu, ${ }^{1,2}$ Mou Chen, ${ }^{1,3}$ and Xiaoming Chen ${ }^{1,3}$ \\ ${ }^{1}$ College of Automation Engineering, Nanjing University of Aeronautics and Astronautics, Nanjing, Jiangsu 210016, China \\ ${ }^{2}$ Nanhang Jincheng College, Nanjing, Jiangsu 211156, China \\ ${ }^{3}$ Jiangsu Key Laboratory of Internet of Things and Control Technologies, Nanjing University of Aeronautics and Astronautics, Nanjing, \\ Jiangsu 210016, China
}

Correspondence should be addressed to Mou Chen; chenmou@nuaa.edu.cn

Received 1 September 2015; Accepted 21 October 2015

Academic Editor: Chenguang Yang

Copyright $(2015$ Bei Wu et al. This is an open access article distributed under the Creative Commons Attribution License, which permits unrestricted use, distribution, and reproduction in any medium, provided the original work is properly cited.

\begin{abstract}
A bounded controller is proposed for a class of uncertain discrete time-delay systems with nonlinearity and disturbance based on state estimator and disturbance observer technique. A state estimator is developed to estimate the unmeasured system state vector. Suppose that the disturbance is generated by an exogenous system; a disturbance observer is designed to estimate the unknown disturbance. The parameters of the state estimator and the disturbance observer are calculated by solving linear matrix inequalities (LMIs). By applying the outputs of the state estimator and the disturbance observer, the sufficient condition for the existence of the bounded controller is derived based on an appropriate Lyapunov function candidate. Under the developed bounded controller, the stability of the closed-loop system can be guaranteed. Simulation examples are provided to show the effectiveness of the proposed bounded control scheme.
\end{abstract}

\section{Introduction}

Time-delay phenomenon widely exists in most of industrial systems, such as biological systems, hydraulic systems, transmission systems, electrical networks, and chemical systems [1-5]. The existence of time-delay leads to performance degradation or even system instability and causes the complication to the analysis and design of the controller. Up to now, many control schemes have been studied for timedelay systems, such as guaranteed cost control [1], $H_{\infty}$ control $[2,4]$, adaptive neural network control $[5,6]$, sliding mode control [7, 8], and disturbance-observer-based control [9-14]. However, most of the above-mentioned research results are dealing with continuous-time systems. Due to the fast development of computers and digital signal processor (DSP) chips, considerable attention has been paid to the study of discrete time-delay systems $[15,16]$. The stability control problem of discrete time-delay systems can be divided into delay-dependent stability control [17-19] and delayindependent stability control [20]. Since delay-dependent conditions are less conservative than delay-independent conditions, more concentrations have been given on the delay dependent stability analysis.

Usually, the model errors, the measurement errors, and the external disturbances inevitably exist in dynamic systems, which can also further cause the degradation of system performance and even instability. The problem of robust stability analysis and robust controller design has been extensively studied for discrete-time systems with uncertainty and/or disturbance. A novel adaptive-critic-based neural network (NN) controller was proposed for nonlinear singleinput-single-output (SISO) discrete-time systems in [21]. The novel adaptive control technique was proposed for discretetime multi-input-multi-output (MIMO) systems in [22]. The improved dynamic surface control design was studied for constrained hypersonic flight models in [23]. In [24], based on the key ideas of "future outputs prediction" and "nearest-neighbour compensation," the adaptive predictive control laws were developed for nonlinear autoregressive moving average systems. In [25], the problem of robust 
stabilization was investigated for discrete-time singular largescale systems with time-invariant norm-bounded uncertainties. Based on the dynamic surface control technique, both indirect and direct global neural controllers were developed for the strict-feedback systems in [26]. In [27], the output feedback adaptive neural network controllers were developed for nonlinear discrete-time systems. The output feedback adaptive control technique was investigated for discrete-time systems with unknown control directions in [28]. In [29], a robust adaptive sliding mode controller was constructed for discrete time-delay systems with mismatched uncertainties and matched external disturbances. Since the disturbances widely exist in practical systems and most of them are difficult to be measured, many advanced control approaches, such as adaptive control and sliding mode control, have been proposed. However, the control approaches mentioned above are rejecting disturbances via feedback control based on the tracking error, which can not deal with strong disturbances directly and fast [30]. Then, the disturbance observer was proposed to estimate unmeasured disturbances for engineering application systems such as direct-current servomotor systems [31], robotic manipulators [32], and data storage systems [33]. A reduced order disturbance observer was studied for discrete-time linear systems in [33]. Based on the disturbance observer, an antidisturbance controller was investigated for discrete time-varying delay systems with multiple disturbances under actuator failures in [34]. In [35], disturbance-observer-based control and $H_{\infty}$ control were discussed for A4D aircraft at a flight condition of $15000 \mathrm{ft}$ altitude and 0.9 Mach, the states were made up with the forward velocity, the angle of attack, the pitching velocity, and the pitching angle, and the state delay was considered. A composite fuzzy control was investigated for uncertain nonlinear systems based on the disturbance observer technique in [36]. However, the input time-delay was seldom considered in the research results mentioned above.

Time delay in control inputs brings new challenges in the controller design. The problem of $H_{\infty}$ control was investigated for discrete time-varying input-delay systems via Riccati difference equation in [37]. In [38], the state feedback prediction-based control law was developed for a discrete-time system with input time-varying delay. Applying Artstein's reduction method and the scaled bounded real lemma, a sufficient condition was presented to stabilize linear discrete-time systems with time-varying input delay and model uncertainties in [39]. In [40, 41], the guaranteed cost control problem was studied for a class of discrete timedelay systems via state feedback. The problem of multiple input delays was discussed in [42]. A robust $H_{\infty}$ state feedback controller was designed for discrete time systems with interval time-varying delays in both states and control inputs in [43]. The state variables mentioned above were required as mensurable. However, not all of the state variables can be measured directly in practical applications. Thus, it is necessary to estimate the unmeasured state variables.

State observer, the product of the development of modern control engineering with the characteristics of good stability and strong robustness, has been widely used in various fields, such as diesel engines [44], stirred tank reactor [45], and permanent magnet synchronous motors [46]. The problem of the state-observer-based finite-time $H_{\infty}$ control was studied for singular stochastic systems using the descriptor system approach in [47]. Based on the future states prediction, adaptive control technique was investigated for a class of discretetime MIMO nonlinear systems in [48]. In [49], a robust delay-dependent state estimator was investigated for a class of discrete-time Bidirectional Associative Memory (BAM) neural networks. A reduced-order Kalman-type observer was designed for nonlinear discrete-time systems in [50]. In [51], a fuzzy observer was proposed for discrete time-delay systems with nonlinearity. An observer-based finite-time $H_{\infty}$ controller was developed for discrete-time Markovian jump nonlinear systems with time delays in [52]. In [53], a state observer was designed for discrete time-delay impulsive switched systems with nonlinearity. A nonlinear observer was proposed for discrete-time uncertain nonlinear systems in [20]. In [54], the problem of reliable observer-based $H_{\infty}$ control was studied for discrete-time Takagi-Sugeno (T-S) fuzzy systems with time-varying delays and stochastic actuator faults. To the best of author's knowledge, the discrete-time system subject to input saturation, parameter uncertainties, nonlinearity, disturbance, and time delays in both the states and the control inputs has not been investigated.

Motivated by the above discussion, we develop a bounded control scheme for uncertain discrete-time nonlinear system with disturbance and time delays in both the states and the control inputs in this paper. A state estimator and a disturbance observer are developed separately to estimate the unknown states and the disturbances generated by an exogenous system. Using the outputs of the state estimator and the disturbance observer, a bounded controller is designed. The Lyapunov function candidate is constructed so that the closed-loop system is asymptotically stable via LMI method. The paper is organized as follows: the problem formulation and preliminaries are given in Section 2. In Section 3, a state estimator and a disturbance observer are presented, respectively, and the bounded controller is designed for the discrete time-delay uncertain systems. Simulation results are given in Section 4, followed by concluding remarks in Section 5.

\section{Problem Formulation and Preliminaries}

Consider the following uncertain discrete-time nonlinear system with disturbance and time delays:

$$
\begin{aligned}
x(k+1)= & \left(G_{0}+\Delta G_{0}\right) x(k)+\left(G_{0 d}+\Delta G_{0 d}\right) x(k-T) \\
& +F_{01} f_{01}(x(k), k) \\
& +H_{0}(u(k-T)+d(k)) \\
y(k)= & C_{0} x(k)
\end{aligned}
$$

where $x(k) \in R^{n}, u(k) \in R^{m}, y(k) \in R^{p}$, and $d(k) \in$ $R^{m}$ are unmeasured state vector, control input, output, and disturbance, respectively. $f_{01}(x(k), k) \in R^{q}$ is the nonlinear function. $G_{0}, G_{0 d}, H_{0}$, and $C_{0}$ are known constant matrices with appropriate dimensions. $\Delta G_{0}$ and $\Delta G_{0 d}$ are parameter 
uncertainties. $F_{01}$ is a corresponding weighting matrix with appropriate dimensions. $T$ is a known constant delay. The system parameter uncertainties are defined as

$$
\left[\begin{array}{ll}
\Delta G_{0} & \Delta G_{0 d}
\end{array}\right]=D \Delta(k)\left[\begin{array}{ll}
M_{1} & M_{2}
\end{array}\right]
$$

where $D, M_{1}$, and $M_{2}$ are known constant matrices and $\Delta(k)$ is an unknown matrix satisfying

$$
\Delta^{T}(k) \Delta(k) \leq I
$$

Here, $I$ denotes the identity matrix with appropriate dimension.

Assume that $\operatorname{sat}(u(k))=\left[\operatorname{sat}\left(u_{1}(k)\right), \ldots, \operatorname{sat}\left(u_{m}(k)\right)\right]^{T}$ is a saturated nonlinear function. Each element $\operatorname{sat}\left(u_{i}(k)\right)$ of $\operatorname{sat}(u(k))$ is defined as

$$
\operatorname{sat}\left(u_{i}(k)\right)=\operatorname{sign}\left(u_{i}(k)\right) \min \left(u_{i \max },\left|u_{i}(k)\right|\right),
$$

where $u_{i \max }$ represents the saturation level of $u_{i}(k)$ and is assumed as known, $i=1, \ldots, m$.

To design the bounded controller for the uncertain discrete time-delay system with nonlinearity and disturbance, the following assumptions and lemmas are needed.

Assumption 1. Assume that state vector $x(k)$ of the discrete time-delay system (1) is unmeasured.

Assumption 2 (see Guo and Chen [55]). Assume that the disturbance $d(k)$ is generated by the following exogenous system:

$$
\begin{aligned}
w(k+1) & =W w(k), \\
d(k) & =V w(k),
\end{aligned}
$$

where $w(k) \in R^{s}, W$, and $V$ are matrices with corresponding dimensions. As shown in $[9,55,56]$, a wide class of disturbances, such as harmonic disturbances, can be described by this model. This model has been extensively used in the disturbance observer design.

Assumption 3 (see Wei et al. [35]). Assume that there exists a constant matrix $U_{01}$ such that

$$
\begin{aligned}
& \left\|f_{01}\left(x_{1}(k), k\right)-f_{01}\left(x_{2}(k), k\right)\right\| \\
& \quad \leq\left\|U_{01}\left(x_{1}(k)-x_{2}(k)\right)\right\|, \quad f_{01}(0, k)=0 .
\end{aligned}
$$

Assumption 4 (see Wei et al. [35]). $\left(G_{0}, H_{0}\right)$ is controllable; $\left(W, H_{0} V\right)$ and $\left(G_{0}, C_{0}\right)$ are observable.

Lemma 5 (see Shi et al. [57]). Given symmetric constant matrices $S_{11}, S_{22}$ and constant matrix $S_{12}$, then $S_{22}<0$ and $S_{11}-S_{12} S_{22}^{-1} S_{12}^{T}<0$ hold if and only if

$$
\left[\begin{array}{ll}
S_{11} & S_{12} \\
S_{12}^{T} & S_{22}
\end{array}\right]<0 .
$$

Lemma 6 (see Zhu et al. [58]). Assume that $Y, A$, and $B$ are real matrices with appropriate dimensions, $Y$ is a symmetric matrix, and $X^{T} X \leq I$. Then

$$
Y+A X B+B^{T} X^{T} A^{T}<0
$$

holds if and only if there exists a scalar $\alpha>0$ such that

$$
Y+\alpha A A^{T}+\alpha^{-1} B^{T} B<0 .
$$

Lemma 7 (see Song and Wang [59]). Assume that $\mathscr{D}=$ $\left\{D_{1}, D_{2}, \ldots, D_{2^{m}}\right\}$ is the set of $m \times m$ diagonal matrices with diagonal elements being either 0 or 1 . If $D_{l} \in \mathscr{D}$, we obtain that $D_{l}^{-}=I-D_{l} \in \mathscr{D}$, where $l=1, \ldots, 2^{m}$.

Lemma 8 (see Zheng and $\mathrm{Wu}[60]$ ). Assume that $u(k) \in R^{m}$, $v(k)=\left[v_{1}, \ldots, v_{m}\right]^{T} \in R^{m}$.If $\left|v_{i}\right| \leq u_{\text {imax }}$, the saturated input $\operatorname{sat}(u(k))$ can be represented as $\operatorname{sat}(u(k))=\sum_{l=1}^{2^{m}} \eta_{l}\left(D_{l} u(k)+\right.$ $\left.D_{l}^{-} v(k)\right)$, where $i=1, \ldots, m, 0 \leq \eta_{l} \leq 1$, and $\sum_{l=1}^{2^{m}} \eta_{l}=1$.

The control objective of this paper is to find a sufficient condition in order to guarantee the stability of the uncertain discrete time-delay system described by (1) under a bounded controller.

\section{Design of Bounded Controller Based on the State Estimator and the Disturbance Observer}

In this section, the state estimator and the disturbance observer are designed to estimate the unmeasured state vector and disturbance of the discrete time-delay uncertain system. The output signals of the disturbance observer and the state estimator are delivered to the bounded controller as its input signals. The stability condition of the closed-loop system is derived by the chosen Lyapunov function as well as LMIs method. The control diagram of the closed-loop system is shown in Figure 1, where $\widehat{x}(k)$ and $\widehat{d}(k)$ are the estimations of $x(k)$ and $d(k)$.

3.1. Design of State Estimator. For the unmeasured state vector in the time-delay uncertain nonlinear system (1), a state estimator is needed to estimate $x(k)$. The state estimator is designed as

$$
\begin{aligned}
\widehat{x}(k+1)= & G_{0} \widehat{x}(k)+G_{0 d} \widehat{x}(k-T)+H_{0} u(k-T) \\
& +H_{0} \widehat{d}(k)+F_{01} f_{01}(\widehat{x}(k), k) \\
& +L_{1}\left(y(k)-C_{0} \widehat{x}(k)\right),
\end{aligned}
$$

where $L_{1}$ is an observer gain matrix which is needed to be designed by the comprehensive consideration between response speed and the sensitivity to disturbance and noise.

Define $\widetilde{x}(k)=x(k)-\widehat{x}(k)$. Considering (1) and (10) yields

$$
\begin{aligned}
\tilde{x}(k+1)= & \left(G_{0}-L_{1} C_{0}\right) \tilde{x}(k)+\Delta G_{0} x(k) \\
& +G_{0 d} \tilde{x}(k-T)+\Delta G_{0 d} x(k-T) \\
& +F_{01} \tau_{01}(k)+H_{0}(d(k)-\widehat{d}(k)),
\end{aligned}
$$

where $\tau_{01}(k)=\left(f_{01}(x(k), k)-f_{01}(\widehat{x}(k), k)\right)$. 


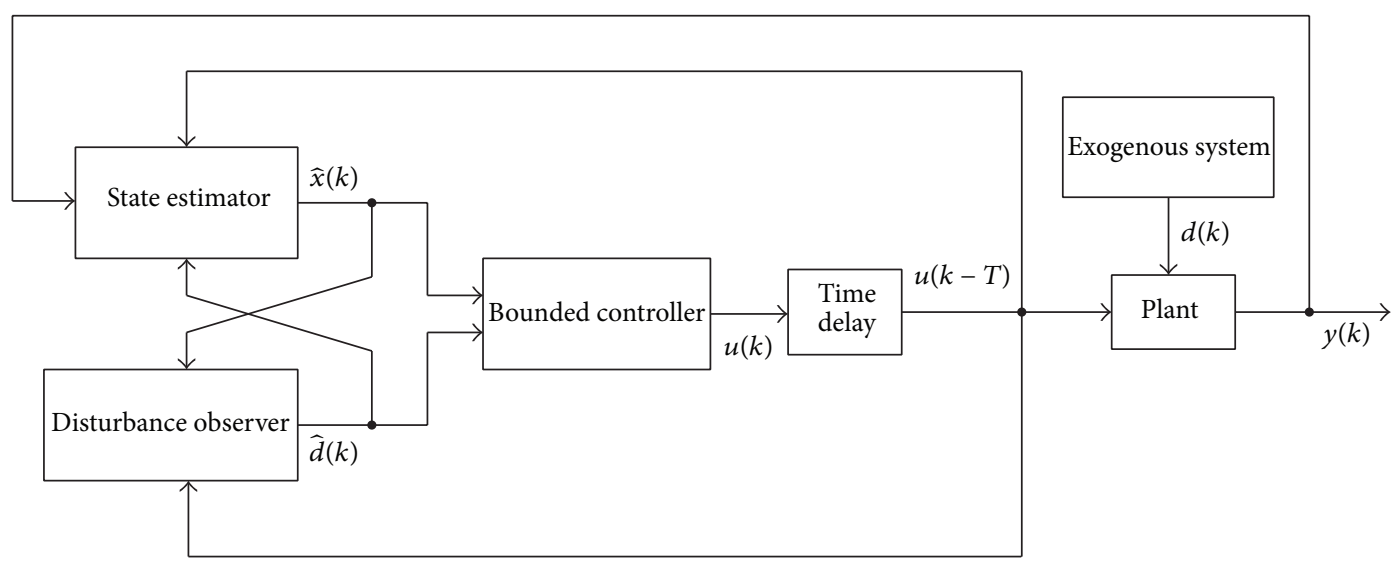

FIGURE 1: The control diagram of system.

3.2. Design of Disturbance Observer. In order to monitor the disturbance of the discrete time-delay uncertain system (1), a disturbance observer is proposed as follows [35]:

$$
\begin{aligned}
& \widehat{d}(k)=V \widehat{w}(k), \\
& \widehat{w}(k)=g(k)-L \widehat{x}(k), \\
& g(k+1)=\left(W+L H_{0} V\right) \widehat{w}(k)+L\left(G_{0} \widehat{x}(k)\right. \\
& \left.\quad+G_{0 d} \widehat{x}(k-T)+F_{01} f_{01}(\widehat{x}(k), k)+H_{0} u(k-T)\right),
\end{aligned}
$$

where $\widehat{w}(k) \in R^{s}$ is the estimation of $w(k)$ and $g(k) \in R^{s}$ is the auxiliary vector as the state of the disturbance observer.

Define

$$
\widetilde{w}(k)=w(k)-\widehat{w}(k)
$$

Considering (5), (13) and invoking (10), we obtain

$$
\widetilde{w}(k+1)=W \widetilde{w}(k)+L_{2} C_{0} \tilde{x}(k),
$$

where $L_{2}=L L_{1}$.

Substituting (5), (12), and (13) into (11) gives

$$
\begin{aligned}
\tilde{x}(k+1)= & \left(G_{0}-L_{1} C_{0}\right) \tilde{x}(k)+\Delta G_{0} x(k) \\
& +G_{0 d} \tilde{x}(k-T)+\Delta G_{0 d} x(k-T) \\
& +F_{01} \tau_{01}(k)+H_{0} V \widetilde{w}(k) .
\end{aligned}
$$

3.3. Design of Bounded Controller. The state estimator and the disturbance observer have been designed to estimate the unmeasured state vector and the unknown disturbance. Based on the output signals of $\widehat{x}(k)$ and $\widehat{d}(k)$, the bounded controller is designed as

$$
u(k)=\operatorname{sat}\left(K_{1} \widehat{x}(k)+K_{2} \widehat{d}(k)\right),
$$

where $K_{1}$ and $K_{2}$ are feedback gain matrices.
The saturated linear region of $u(k)$ is defined as

$$
\begin{aligned}
\mathscr{L} & \left(V_{1}, V_{2}\right)=\{(\widehat{x}(k), \widehat{d}(k)) \\
& \in R^{n+m}:\left|V_{i 1} \widehat{x}(k)+V_{i 2} \widehat{d}(k)\right| \leq u_{i \max }, i \\
& =1, \ldots, m\},
\end{aligned}
$$

where $V_{1}=\left[V_{11}, \ldots, V_{m 1}\right]^{T} \in R^{m \times n}, V_{i 1} \in R^{1 \times n}, V_{2}=$ $\left[V_{12}, \ldots, V_{m 2}\right]^{T} \in R^{m \times m}, V_{i 2} \in R^{1 \times m}$, and $i=1, \ldots, m$. Using Lemma 8, (16) can be rewritten as

$$
\begin{gathered}
u(k)=\sum_{l=1}^{2^{m}} \eta_{l}\left(D_{l}\left(K_{1} \widehat{x}(k)+K_{2} \widehat{d}(k)\right)\right. \\
\left.+D_{l}^{-}\left(V_{1} \hat{x}(k)+V_{2} \widehat{d}(k)\right)\right) .
\end{gathered}
$$

Substituting (12) and (18) into (1), the closed-loop system is given by

$$
\begin{aligned}
& x(k+1)=\sum_{l=1}^{2^{m}} \eta_{l}\left\{\left(G_{0}+\Delta G_{0}\right) x(k)\right. \\
& \left.+\left(\bar{G}_{d}+\Delta G_{0 d}\right) x(k-T)+H_{0} V w(k)\right\} \\
& +\sum_{l=1}^{2^{m}} \eta_{l}\left\{F_{01} f_{01}(x(k), k)+\bar{W} w(k-T)\right. \\
& -\bar{K} \tilde{x}(k-T)-\bar{W} \widetilde{w}(k-T)\},
\end{aligned}
$$

where $\bar{G}_{d}=G_{0 d}+H_{0}\left(D_{l} K_{1}+D_{l}^{-} V_{1}\right), \bar{W}=H_{0}\left(D_{l} K_{2}+D_{l}^{-} V_{2}\right) V$, and $\bar{K}=H_{0}\left(D_{l} K_{1}+D_{l}^{-} V_{1}\right)$.

Combining (14), (15), and (19), the whole closed-loop system is expressed as

$$
\begin{aligned}
& \bar{x}(k+1)=\sum_{l=1}^{2^{m}} \eta_{l}\left\{G \bar{x}(k)+G_{d} \bar{x}(k-T)\right. \\
& +F f(\bar{x}(k), k)+H w(k)+M w(k-T)+E \tau(k)\},
\end{aligned}
$$


where

$$
\begin{aligned}
& \bar{x}(k)=\left[\begin{array}{c}
x(k) \\
\widetilde{w}(k) \\
\widetilde{x}(k)
\end{array}\right], \\
& \bar{x}(k-T)=\left[\begin{array}{c}
x(k-T) \\
\widetilde{w}(k-T) \\
\tilde{x}(k-T)
\end{array}\right], \\
& F=\left[\begin{array}{c}
F_{01} \\
0 \\
0
\end{array}\right], \\
& G=\left[\begin{array}{ccc}
G_{0}+\Delta G_{0} & 0 & 0 \\
0 & W & L_{2} C_{0} \\
\Delta G_{0} & H_{0} V & G_{0}-L_{1} C_{0}
\end{array}\right] \text {, } \\
& H=\left[\begin{array}{c}
H_{0} V \\
0 \\
0
\end{array}\right] \text {, } \\
& G_{d}=\left[\begin{array}{ccc}
\bar{G}_{d}+\Delta G_{0 d} & -\bar{W} & -\bar{K} \\
0 & 0 & 0 \\
\Delta G_{0 d} & 0 & G_{0 d}
\end{array}\right] \text {, } \\
& M=\left[\begin{array}{c}
\bar{W} \\
0 \\
0
\end{array}\right] \text {, } \\
& E=\left[\begin{array}{c}
0 \\
0 \\
F_{01}
\end{array}\right], \\
& \tau(k)=\tau_{01}(k), \\
& f(\bar{x}(k), k)=f_{01}(x(k), k) \text {. }
\end{aligned}
$$

According to Assumption 3, we obtain

$$
\begin{aligned}
\|f(\bar{x}(k), k)\| & =\left\|f_{01}(x(k), k)\right\| \leq\left\|U_{01} x(k)\right\| \\
& =\left\|U_{1} \bar{x}(k)\right\|, \\
\|\tau(k)\| & \leq\left\|U_{01}(x(k)-\widehat{x}(k))\right\|=\left\|U_{2} \bar{x}(k)\right\|,
\end{aligned}
$$

where $U_{1}=\left[\begin{array}{lll}U_{01} & 0 & 0\end{array}\right] \in R^{q \times(2 n+s)}$ and $U_{2}=\left[\begin{array}{lll}0 & 0 & U_{01}\end{array}\right] \epsilon$ $R^{q \times(2 n+s)}$.

Disturbance gain matrix $L$, state estimator gain matrix $L_{1}$, and feedback gain matrices $K_{1}, K_{2}, V_{1}$, and $V_{2}$ play an important role in closed-loop system stability and estimation error stability. The values of these matrices can be derived by solving LMIs. The stability condition of the closed-loop system (20) is given in the following theorem.
Theorem 9. For given scalars $\varepsilon>0, \lambda_{1}>0$, and $\lambda_{2}>0$, the closed-loop system (20) is asymptotically stable if there exist symmetric positive-defined matrices $Q_{11}=P_{11}^{-1}>0, P_{22}>0$, $P_{33}>0, N_{11}=S_{11}^{-1}>0, S_{22}>0$, and $S_{33}>0$ and matrices $Z_{1}, Z_{2}, Z_{3}, R_{1}, R_{2}, R_{3}, R_{4}, K_{1}, K_{2}, V_{1}$, and $V_{2}$ such that the following matrix inequality holds:

$$
N_{6}=\left[\begin{array}{llc}
\Pi_{11} & * & * \\
\Pi_{21} & \Pi_{22} & * \\
\Pi_{31} & \Pi_{32} & \Pi_{33}
\end{array}\right]<0
$$

where

$$
\begin{aligned}
& \Pi_{11} \\
& =\left[\begin{array}{ccccccc}
-Q_{11} & * & * & * & * & * & * \\
0 & -P_{22}+S_{22} & * & * & * & * & * \\
0 & 0 & -P_{33}+S_{33} & * & * & * & * \\
0 & 0 & 0 & -N_{11} & * & * & * \\
0 & 0 & 0 & 0 & -S_{22} & * & * \\
0 & 0 & 0 & 0 & 0 & -S_{33} & * \\
0 & 0 & 0 & 0 & 0 & 0 & -\lambda_{1}^{2} I
\end{array}\right],
\end{aligned}
$$

$\Pi_{21}$

$$
=\left[\begin{array}{ccccccc}
0 & 0 & 0 & 0 & 0 & 0 & 0 \\
0 & 0 & 0 & 0 & 0 & 0 & 0 \\
0 & 0 & 0 & 0 & 0 & 0 & 0 \\
0 & 0 & 0 & 0 & 0 & 0 & 0 \\
G_{0} Q_{11} & 0 & 0 & G^{\prime} & -\bar{W} & -\bar{K} & F_{01} \\
0 & P_{22} W & R_{2} C_{0} & 0 & 0 & 0 & 0 \\
0 & P_{33} H_{0} V & P_{33} G_{0}-R_{1} C_{0} & 0 & 0 & P_{33} G_{0 d} & 0
\end{array}\right],
$$

$\Pi_{22}$

$$
=\left[\begin{array}{ccccccc}
-\lambda_{2}^{2} I & * & * & * & * & * & * \\
0 & -Z_{1}-Z_{1}^{T} & * & * & * & * & * \\
0 & Z_{1}-Z_{2}^{T} & Z_{2}+Z_{2}^{T} & * & * & * & * \\
0 & Z_{1}-Z_{3}^{T} & Z_{2}+Z_{3}^{T} & Z_{3}+Z_{3}^{T} & * & * & * \\
0 & H_{0} V & \bar{W} & 0 & -Q_{11} & * & * \\
0 & 0 & 0 & 0 & 0 & -P_{22} & * \\
P_{33} F_{01} & 0 & 0 & 0 & 0 & 0 & -P_{33}
\end{array}\right] \text {, }
$$$$
\Pi_{31}=\left[\begin{array}{ccccccc}
U_{01} Q_{11} & 0 & 0 & 0 & 0 & 0 & 0 \\
0 & 0 & U_{01} & 0 & 0 & 0 & 0 \\
0 & 0 & 0 & 0 & 0 & 0 & 0 \\
M_{1} Q_{11} & 0 & 0 & M_{2} N_{11} & 0 & 0 & 0 \\
Q_{11} & 0 & 0 & 0 & 0 & 0 & 0
\end{array}\right],
$$$$
\Pi_{32}=\left[\begin{array}{ccccccc}
0 & 0 & 0 & 0 & 0 & 0 & 0 \\
0 & 0 & 0 & 0 & 0 & 0 & 0 \\
0 & 0 & 0 & 0 & D^{T} & 0 & D^{T} P_{33} \\
0 & 0 & 0 & 0 & 0 & 0 & 0 \\
0 & 0 & 0 & 0 & 0 & 0 & 0
\end{array}\right] \text {, }
$$ 


$$
\Pi_{33}=\left[\begin{array}{ccccc}
-\lambda_{1}^{-2} I & * & * & * & * \\
0 & -\lambda_{2}^{-2} I & * & * & * \\
0 & 0 & -\varepsilon^{-1} I & * & * \\
0 & 0 & 0 & -\varepsilon I & * \\
0 & 0 & 0 & 0 & -N_{11}
\end{array}\right] \text {, }
$$

and $G^{\prime}=G_{0 d} N_{11}+H_{0} D_{l} R_{3}+H_{0} D_{l}^{-} R_{4}, L_{1}=P_{33}^{-1} R_{1}, L_{2}=$ $P_{22}^{-1} R_{2}, K_{1}=R_{3} N_{11}^{-1}$, and $V_{1}=R_{4} N_{11}^{-1}$. * denotes the transposed elements in the symmetric matrix.

Proof. Choose the Lyapunov function as

$$
\begin{aligned}
V(k)= & \bar{x}^{T}(k) P_{1} \bar{x}(k) \\
& +\lambda_{1}^{2} \sum_{i=1}^{k-1}\left[\left\|U_{1} \bar{x}(i)\right\|^{2}-\|f(\bar{x}(i), i)\|^{2}\right] \\
& +\sum_{i=k-T}^{k-1} \bar{x}^{T}(i) S_{1} \bar{x}(i) \\
& +\lambda_{2}^{2} \sum_{i=1}^{k-1}\left[\left\|U_{2} \bar{x}(i)\right\|^{2}-\|\tau(i)\|^{2}\right],
\end{aligned}
$$

where

$$
\begin{aligned}
& P_{1}=\left[\begin{array}{ccc}
P_{11} & 0 & 0 \\
0 & P_{22} & 0 \\
0 & 0 & P_{33}
\end{array}\right]=\left[\begin{array}{ccc}
Q_{11}^{-1} & 0 & 0 \\
0 & P_{22} & 0 \\
0 & 0 & P_{33}
\end{array}\right]>0, \\
& S_{1}=\left[\begin{array}{ccc}
S_{11} & 0 & 0 \\
0 & S_{22} & 0 \\
0 & 0 & S_{33}
\end{array}\right]=\left[\begin{array}{ccc}
N_{11}^{-1} & 0 & 0 \\
0 & S_{22} & 0 \\
0 & 0 & S_{33}
\end{array}\right]>0 .
\end{aligned}
$$

Define $r(j)=w(j+1)-w(j)$. Then, we have

$$
\begin{aligned}
w(k+1) & =w(k)+r(k), \\
w(k)-w(k-T)-\sum_{j=k-T}^{k-1} r(j) & =0 .
\end{aligned}
$$

Denote that

$$
\begin{aligned}
& \Gamma_{1}=-w(k)+w(k-T)+\sum_{j=k-T}^{k-1} r(j), \\
& \Gamma_{2}=Z_{1} w(k)+Z_{2} w(k-T)+Z_{3} \sum_{j=k-T}^{k-1} r(j) .
\end{aligned}
$$

Taking the forward difference of $V(k)$ along the trajectories of system (20), we have

$$
\begin{aligned}
\Delta V & (k)=V(k+1)-V(k)=V(k+1)-V(k) \\
& +\Gamma_{1}^{T} \Gamma_{2}+\Gamma_{2}^{T} \Gamma_{1}=\sum_{l=1}^{2^{m}} \eta_{l}\left\{\bar{x}^{T}(k)\right. \\
& \cdot\left(-P_{1}+S_{1}+\lambda_{1}^{2} U_{1}^{T} U_{1}+\lambda_{2}^{2} U_{2}^{T} U_{2}+G^{T} P_{1} G\right) \bar{x}(k) \\
& +2 \bar{x}^{T}(k-T) G_{d}^{T} P_{1} G \bar{x}(k)+\bar{x}^{T}(k-T) \\
& \cdot\left(G_{d}^{T} P_{1} G_{d}-S_{1}\right) \bar{x}(k-T)+2 f^{T}(\bar{x}(k), k) \\
& \cdot F^{T} P_{1} G \bar{x}(k)+2 f^{T}(\bar{x}(k), k) F^{T} P_{1} G_{d} \bar{x}(k-T) \\
& +f^{T}(\bar{x}(k), k)\left(F^{T} P_{1} F-\lambda_{1}^{2} I\right) f(\bar{x}(k), k) \\
& +2 \tau^{T}(k) E^{T} P_{1} G \bar{x}(k)+2 \tau^{T}(k) E^{T} P_{1} G_{d} \bar{x}(k-T) \\
& +2 \tau^{T}(k) E^{T} P_{1} F f(\bar{x}(k), k)+\tau^{T}(k) \\
& +\left(E^{T} P_{1} E-\lambda_{2}^{2} I\right) \tau(k)+2 w^{T}(k) H^{T} P_{1} G \bar{x}(k) \\
+ & 2 w^{T}(k) H^{T} P_{1} G_{d} \bar{x}(k-T)+2 w^{T}(k) \\
& +H^{T} P_{1} F f(\bar{x}(k), k)+2 w^{T}(k) H^{T} P_{1} E \tau(k)
\end{aligned}
$$$$
+w^{T}(k)\left(H^{T} P_{1} H-Z_{1}-Z_{1}^{T}\right) w(k)
$$$$
+2 w^{T}(k-T) M^{T} P_{1} G \bar{x}(k)+2 w^{T}(k-T)
$$$$
\cdot M^{T} P_{1} G_{d} \bar{x}(k-T)+2 w^{T}(k-T)
$$$$
\cdot M^{T} P_{1} F f(\bar{x}(k), k)+2 w^{T}(k-T) M^{T} P_{1} E \tau(k)
$$$$
+2 w^{T}(k-T)\left(M^{T} P_{1} H+Z_{1}-Z_{2}^{T}\right) w(k)
$$$$
+w^{T}(k-T)\left(M^{T} P_{1} M+Z_{2}+Z_{2}^{T}\right) w(k-T)
$$$$
+2 \sum_{j=k-T}^{k-1} r^{T}(j)\left(Z_{1}-Z_{3}^{T}\right) w(k)
$$$$
+2 \sum_{j=k-T}^{k-1} r^{T}(j)\left(Z_{2}+Z_{3}^{T}\right) w(k-T)
$$$$
\left.+\sum_{j=k-T}^{k-1} r^{T}(j)\left(Z_{3}+Z_{3}^{T}\right) \sum_{j=k-T}^{k-1} r^{T}(j)\right\}
$$$$
\leq \max _{l \in\left[1,2^{m}\right]}\left\{\bar{x}^{T}(k)\right.
$$

$$
\begin{aligned}
& \cdot\left(-P_{1}+S_{1}+\lambda_{1}^{2} U_{1}^{T} U_{1}+\lambda_{2}^{2} U_{2}^{T} U_{2}+G^{T} P_{1} G\right) \bar{x}(k) \\
& +2 \bar{x}^{T}(k-T) G_{d}^{T} P_{1} G \bar{x}(k)+\bar{x}^{T}(k-T)
\end{aligned}
$$




$$
\begin{aligned}
& \cdot\left(G_{d}^{T} P_{1} G_{d}-S_{1}\right) \bar{x}(k-T)+2 f^{T}(\bar{x}(k), k) \\
& \cdot F^{T} P_{1} G \bar{x}(k)+2 f^{T}(\bar{x}(k), k) F^{T} P_{1} G_{d} \bar{x}(k-T) \\
& +f^{T}(\bar{x}(k), k)\left(F^{T} P_{1} F-\lambda_{1}^{2} I\right) f(\bar{x}(k), k) \\
& +2 \tau^{T}(k) E^{T} P_{1} G \bar{x}(k)+2 \tau^{T}(k) E^{T} P_{1} G_{d} \bar{x}(k-T) \\
& +2 \tau^{T}(k) E^{T} P_{1} F f(\bar{x}(k), k)+\tau^{T}(k) \\
& \cdot\left(E^{T} P_{1} E-\lambda_{2}^{2} I\right) \tau(k)+2 w^{T}(k) H^{T} P_{1} G \bar{x}(k) \\
& +2 w^{T}(k) H^{T} P_{1} G_{d} \bar{x}(k-T)+2 w^{T}(k) \\
& \cdot H^{T} P_{1} F f(\bar{x}(k), k)+2 w^{T}(k) H^{T} P_{1} E \tau(k) \\
& +w^{T}(k)\left(H^{T} P_{1} H-Z_{1}-Z_{1}^{T}\right) w(k) \\
& +2 w^{T}(k-T) M^{T} P_{1} G \bar{x}(k)+2 w^{T}(k-T) \\
& \cdot M^{T} P_{1} G_{d} \bar{x}(k-T)+2 w^{T}(k-T) \\
& \cdot M^{T} P_{1} F f(\bar{x}(k), k)+2 w^{T}(k-T) M^{T} P_{1} E \tau(k) \\
& +2 w^{T}(k-T)\left(M^{T} P_{1} H+Z_{1}-Z_{2}^{T}\right) w(k) \\
& +w^{T}(k-T)\left(M^{T} P_{1} M+Z_{2}+Z_{2}^{T}\right) w(k-T)
\end{aligned}
$$

$$
\left.\begin{array}{l}
+2 \sum_{j=k-T}^{k-1} r^{T}(j)\left(Z_{1}-Z_{3}^{T}\right) w(k) \\
+2 \sum_{j=k-T}^{k-1} r^{T}(j)\left(Z_{2}+Z_{3}^{T}\right) w(k-T) \\
+\sum_{j=k-T}^{k-1} r^{T}(j)\left(Z_{3}+Z_{3}^{T}\right) \sum_{j=k-T}^{k-1} r^{T}(j)
\end{array}\right\} .
$$

Then, (29) can be rewritten as

$$
\Delta V(k) \leq \max _{l \in\left[1,2^{m}\right]}\left[\begin{array}{c}
\bar{x}(k) \\
\bar{x}(k-T) \\
f(\bar{x}(k), k) \\
\tau(k) \\
w(k) \\
\sum_{j=k-T}^{k-1} r(j)
\end{array}\right]^{T} N_{1}\left[\begin{array}{c}
\bar{x}(k) \\
\bar{x}(k-T) \\
f(\bar{x}(k), k) \\
\tau(k) \\
w(k) \\
w(k-T) \\
\sum_{j=k-T}^{k-1} r(j)
\end{array}\right],
$$

where

$$
\begin{aligned}
& N_{1}=\left[\begin{array}{ccccccc}
\Pi_{1} & * & * & * & * & * & * \\
G_{d}^{T} P_{1} G & \Pi_{2} & * & * & * & * & * \\
F^{T} P_{1} G & F^{T} P_{1} G_{d} & \Pi_{3} & * & * & * & * \\
E^{T} P_{1} G & E^{T} P_{1} G_{d} & E^{T} P_{1} F & \Pi_{4} & * & * & * \\
H^{T} P_{1} G & H^{T} P_{1} G_{d} & H^{T} P_{1} F & H^{T} P_{1} E & \Pi_{5} & * & * \\
M^{T} P_{1} G & M^{T} P_{1} G_{d} & M^{T} P_{1} F & M^{T} P_{1} E & \Pi_{8} & \Pi_{6} & * \\
0 & 0 & 0 & 0 & Z_{1}-Z_{3}^{T} & Z_{2}+Z_{3}^{T} & \Pi_{7}
\end{array}\right] \text {, } \\
& \Pi_{1}=-P_{1}+S_{1}+\lambda_{1}^{2} U_{1}^{T} U_{1}+\lambda_{2}^{2} U_{2}^{T} U_{2}+G^{T} P_{1} G, \\
& \Pi_{2}=G_{d}^{T} P_{1} G_{d}-S_{1}, \\
& \Pi_{3}=F^{T} P_{1} F-\lambda_{1}^{2} I, \\
& \Pi_{4}=E^{T} P_{1} E-\lambda_{2}^{2} I, \\
& \Pi_{5}=H^{T} P_{1} H-Z_{1}-Z_{1}^{T} \text {, } \\
& \Pi_{6}=M^{T} P_{1} M+Z_{2}+Z_{2}^{T} \text {, } \\
& \Pi_{7}=Z_{3}+Z_{3}^{T} \\
& \Pi_{8}=M^{T} P_{1} H+Z_{1}-Z_{2}^{T} .
\end{aligned}
$$


If $N_{1}$ can be proved to be less than 0 , we have $\Delta V(k)<$ 0 . Then, the closed-loop system (20) is asymptotically stable. Applying Schur complement theorem, $N_{1}<0$ if and only if

$$
N_{2}=\left[\begin{array}{cc}
\Gamma_{11} & * \\
\Gamma_{21} & \Gamma_{22}
\end{array}\right]<0
$$

where

$$
\begin{aligned}
& \Gamma_{11}=\left[\begin{array}{ccccccc}
-P_{1}+S_{1} & * & * & * & * & * & * \\
0 & -S_{1} & * & * & * & * & * \\
0 & 0 & -\lambda_{1}^{2} I & * & * & * & * \\
0 & 0 & 0 & -\lambda_{2}^{2} I & * & * & * \\
0 & 0 & 0 & 0 & -Z_{1}-Z_{1}^{T} & * & * \\
0 & 0 & 0 & 0 & Z_{1}-Z_{2}^{T} & Z_{2}+Z_{2}^{T} & * \\
0 & 0 & 0 & 0 & Z_{1}-Z_{3}^{T} & Z_{2}+Z_{3}^{T} & Z_{3}+Z_{3}^{T}
\end{array}\right] \text {, } \\
& \Gamma_{21}=\left[\begin{array}{ccccccc}
G & G_{d} & F & E & H & M & 0 \\
U_{1} & 0 & 0 & 0 & 0 & 0 & 0 \\
U_{2} & 0 & 0 & 0 & 0 & 0 & 0
\end{array}\right] \text {, } \\
& \Gamma_{22}=\left[\begin{array}{ccc}
-P_{1}^{-1} & * & * \\
0 & -\lambda_{1}^{-2} I & * \\
0 & 0 & -\lambda_{2}^{-2} I
\end{array}\right] \text {. }
\end{aligned}
$$

Substituting (21) and (26) into (32) yields

$$
N_{3}=\left[\begin{array}{ccc}
\Lambda_{11} & * & * \\
\Lambda_{21} & \Lambda_{22} & * \\
\Lambda_{31} & \Lambda_{32} & \Lambda_{33}
\end{array}\right],
$$

where

$$
\begin{aligned}
& \Lambda_{11}=\left[\begin{array}{ccccccc}
-P_{11}+S_{11} & * & * & * & * & * & * \\
0 & -P_{22}+S_{22} & * & * & * & * & * \\
0 & 0 & -P_{33}+S_{33} & * & * & * & * \\
0 & 0 & 0 & -S_{11} & * & * & * \\
0 & 0 & 0 & 0 & -S_{22} & * & * \\
0 & 0 & 0 & 0 & 0 & -S_{33} & * \\
0 & 0 & 0 & 0 & 0 & 0 & -\lambda_{1}^{2} I
\end{array}\right] \text {, } \\
& \Lambda_{21}=\left[\begin{array}{ccccccc}
0 & 0 & 0 & 0 & 0 & 0 & 0 \\
0 & 0 & 0 & 0 & 0 & 0 & 0 \\
0 & 0 & 0 & 0 & 0 & 0 & 0 \\
0 & 0 & 0 & 0 & 0 & 0 & 0 \\
G_{0}+\Delta G_{0} & 0 & 0 & \bar{G}_{d}+\Delta G_{0 d} & -\bar{W} & -\bar{K} & F_{01} \\
0 & W & L_{2} C_{0} & 0 & 0 & 0 & 0 \\
\Delta G_{0} & H_{0} V & G_{0}-L_{1} C_{0} & \Delta G_{0 d} & 0 & G_{0 d} & 0
\end{array}\right] \text {, }
\end{aligned}
$$




$$
\begin{aligned}
& \Lambda_{22}=\left[\begin{array}{ccccccc}
-\lambda_{2}^{2} I & * & * & * & * & * & * \\
0 & -Z_{1}-Z_{1}^{T} & * & * & * & * & * \\
0 & Z_{1}-Z_{2}^{T} & Z_{2}+Z_{2}^{T} & * & * & * & * \\
0 & Z_{1}-Z_{3}^{T} & Z_{2}+Z_{3}^{T} & Z_{3}+Z_{3}^{T} & * & * & * \\
0 & H_{0} V & \bar{W} & 0 & -Q_{11} & * & * \\
0 & 0 & 0 & 0 & 0 & -P_{22}^{-1} & * \\
F_{01} & 0 & 0 & 0 & 0 & 0 & -P_{33}^{-1}
\end{array}\right] \text {, } \\
& \Lambda_{31}=\left[\begin{array}{ccccccc}
U_{01} & 0 & 0 & 0 & 0 & 0 & 0 \\
0 & 0 & U_{01} & 0 & 0 & 0 & 0
\end{array}\right] \text {, } \\
& \Lambda_{32}=\left[\begin{array}{lllllll}
0 & 0 & 0 & 0 & 0 & 0 & 0 \\
0 & 0 & 0 & 0 & 0 & 0 & 0
\end{array}\right] \text {, } \\
& \Lambda_{33}=\left[\begin{array}{cc}
-\lambda_{1}^{-2} I & * \\
0 & -\lambda_{2}^{-2} I
\end{array}\right] \text {. }
\end{aligned}
$$

Using Lemmas 5 and $6, N_{3}<0$ if and only if

$$
N_{4}=\left[\begin{array}{ccc}
\Lambda_{11} & * & * \\
\Lambda_{21}^{\prime} & \Lambda_{22} & * \\
\Lambda_{31}^{\prime} & \Lambda_{32}^{\prime} & \Lambda_{33}^{\prime}
\end{array}\right]<0,
$$

where

$$
\begin{aligned}
\Lambda_{21}^{\prime} & =\left[\begin{array}{ccccccc}
0 & 0 & 0 & 0 & 0 & 0 & 0 \\
0 & 0 & 0 & 0 & 0 & 0 & 0 \\
0 & 0 & 0 & 0 & 0 & 0 & 0 \\
0 & 0 & 0 & 0 & 0 & 0 & 0 \\
G_{0} & 0 & 0 & \bar{G}_{d} & -\bar{W} & -\bar{K} & F_{01} \\
0 & W & L_{2} C_{0} & 0 & 0 & 0 & 0 \\
0 & H_{0} V & G_{0}-L_{1} C_{0} & 0 & 0 & G_{0 d} & 0
\end{array}\right], \\
\Lambda_{31}^{\prime} & =\left[\begin{array}{ccccccc}
U_{01} & 0 & 0 & 0 & 0 & 0 & 0 \\
0 & 0 & U_{01} & 0 & 0 & 0 & 0 \\
0 & 0 & 0 & 0 & 0 & 0 & 0 \\
M_{1} & 0 & 0 & M_{2} & 0 & 0 & 0
\end{array}\right], \\
\Lambda_{32}^{\prime} & =\left[\begin{array}{ccccccc}
0 & 0 & 0 & 0 & 0 & 0 & 0 \\
0 & 0 & 0 & 0 & 0 & 0 & 0 \\
0 & 0 & 0 & 0 & D^{T} & 0 & D^{T} \\
0 & 0 & 0 & 0 & 0 & 0 & 0
\end{array}\right], \\
\Lambda_{33}^{\prime} & =\left[\begin{array}{cccccc}
-\lambda_{1}^{-2} I & * & * & * \\
0 & -\lambda_{2}^{-2} I & * & * \\
0 & 0 & 0 & -\varepsilon^{-1} I & * \\
0 & 0 & 0 & 0 & -\varepsilon I
\end{array}\right] .
\end{aligned}
$$

Left and right multiplying both sides of (36) by $\operatorname{diag}\left\{Q_{11}, I, I, I, I, I, I, I, I, I, I, I, I, I, I, I, I, I\right\}$ and using Schur complement theorem, we have

$$
N_{5}=\left[\begin{array}{ccc}
\Xi_{11} & * & * \\
\Xi_{21} & \Lambda_{22} & * \\
\Xi_{31} & \Xi_{32} & \Xi_{33}
\end{array}\right],
$$

where

$\Xi_{11}$

$=\left[\begin{array}{ccccccc}-Q_{11} & * & * & * & * & * & * \\ 0 & -P_{22}+S_{22} & * & * & * & * & * \\ 0 & 0 & -P_{33}+S_{33} & * & * & * & * \\ 0 & 0 & 0 & -S_{11} & * & * & * \\ 0 & 0 & 0 & 0 & -S_{22} & * & * \\ 0 & 0 & 0 & 0 & 0 & -S_{33} & * \\ 0 & 0 & 0 & 0 & 0 & 0 & -\lambda_{1}^{2} I\end{array}\right]$,
$\Xi_{21}=\left[\begin{array}{cccccccc}0 & 0 & 0 & 0 & 0 & 0 & 0 \\ 0 & 0 & 0 & 0 & 0 & 0 & 0 \\ 0 & 0 & 0 & 0 & 0 & 0 & 0 \\ 0 & 0 & 0 & 0 & 0 & 0 & 0 \\ G_{0} Q_{11} & 0 & 0 & \bar{G}_{d} & -\bar{W} & -\bar{K} & F_{01} \\ 0 & W & L_{2} C_{0} & 0 & 0 & 0 & 0 \\ 0 & H_{0} V & G_{0}-L_{1} C_{0} & 0 & 0 & G_{0 d} & 0\end{array}\right]$,

$\Xi_{31}=\left[\begin{array}{ccccccc}U_{01} Q_{11} & 0 & 0 & 0 & 0 & 0 & 0 \\ 0 & 0 & U_{01} & 0 & 0 & 0 & 0 \\ 0 & 0 & 0 & 0 & 0 & 0 & 0 \\ M_{1} Q_{11} & 0 & 0 & M_{2} & 0 & 0 & 0 \\ Q_{11} & 0 & 0 & 0 & 0 & 0 & 0\end{array}\right]$, 


$$
\begin{aligned}
& \Xi_{32}=\left[\begin{array}{llllccc}
0 & 0 & 0 & 0 & 0 & 0 & 0 \\
0 & 0 & 0 & 0 & 0 & 0 & 0 \\
0 & 0 & 0 & 0 & D^{T} & 0 & D^{T} \\
0 & 0 & 0 & 0 & 0 & 0 & 0 \\
0 & 0 & 0 & 0 & 0 & 0 & 0
\end{array}\right], \\
& \Xi_{33}=\left[\begin{array}{cccccc}
-\lambda_{1}^{-2} I & * & * & * & * \\
0 & -\lambda_{2}^{-2} I & * & * & * \\
0 & 0 & -\varepsilon^{-1} I & * & * \\
0 & 0 & 0 & -\varepsilon I & * \\
0 & 0 & 0 & 0 & -N_{11}
\end{array}\right] .
\end{aligned}
$$

Left and right multiplying both sides of (38) by $\operatorname{diag}\left\{I, I, I, N_{11}, I, I, I, I, I, I, I, I, P_{22}, P_{33}, I, I, I, I, I\right\}$, we obtain the matrix $N_{6}$ of (23). Because $N_{6}<0$, we have $\Delta V(k)<0$, which implies that $x(k) \rightarrow 0, \widetilde{w}(k) \rightarrow 0$, and $\tilde{x}(k) \rightarrow 0$ as $k \rightarrow \infty$. Namely, the closed-loop system (20) is asymptotically stable. This concludes the proof.

\section{Simulation}

In order to verify the correctness of the proposed method, simulation examples are given in the following.

4.1. The Case for Two-Dimension System. Consider the uncertain nonlinear discrete time system (1) with disturbance and time delays in both the states and the control inputs with

$$
\begin{aligned}
G_{0} & =\left[\begin{array}{ll}
0.6 & 0.8 \\
0 & 0.5
\end{array}\right], \\
G_{0 d} & =\left[\begin{array}{ll}
0.2 & 0.1 \\
0 & 0.1
\end{array}\right], \\
D & =\left[\begin{array}{l}
0.1 \\
0.2
\end{array}\right], \\
H_{0} & =\left[\begin{array}{cc}
0.01 & 0 \\
0 & 0.01
\end{array}\right], \\
M_{1} & =\left[\begin{array}{c}
0.2 \\
0.3
\end{array}\right]^{T}, \\
M_{2} & =\left[\begin{array}{c}
0 \\
0.1
\end{array}\right]^{T}, \\
F_{01} & =\left[\begin{array}{c}
0.15 \\
0
\end{array}\right]^{T}, \\
\left.f_{01}(k), k\right) & =\sin \left(3 x_{2}(k)\right) .
\end{aligned}
$$

The parameter matrices of exogenous system (5) are chosen as

$$
\begin{aligned}
& W=\left[\begin{array}{cc}
0.9998 & 0.02 \\
-0.02 & 0.9998
\end{array}\right], \\
& V=\left[\begin{array}{ll}
1 & 0 \\
0 & 1
\end{array}\right] .
\end{aligned}
$$

Suppose that the initial values of state variable are $x(0)=$ $\left[\begin{array}{ll}-2 & 2\end{array}\right]^{T}$, the initial values of state estimator are $\widehat{x}(0)=$ $\left[\begin{array}{ll}-2.001 & 2.001\end{array}\right]^{T}$, the initial values of exogenous inputs are $w(0)=\left[\begin{array}{ll}0.21 & -0.22\end{array}\right]^{T}$, and $\widehat{w}(0)=\left[\begin{array}{ll}0.2 & -0.2\end{array}\right]^{T}$. Choosing $U_{01}=\operatorname{diag}\left\{\begin{array}{ll}0 & 0.2\end{array}\right\}, T=1, u_{1 \max }=0.03, u_{2 \max }=0.1$, $\lambda_{1}=0.1, \lambda_{2}=0.5$, and $\varepsilon=10$, by solving (23), we obtain

$$
\begin{aligned}
K_{1} & =\left[\begin{array}{ll}
-0.0038 & 0.0006 \\
0.0006 & 0.1235
\end{array}\right], \\
K_{2} & =\left[\begin{array}{ll}
-0.0502 & -0.1198 \\
-0.1198 & -0.3544
\end{array}\right], \\
L_{1} & =\left[\begin{array}{ll}
0.5171 & 0.4620 \\
0.0802 & 0.5118
\end{array}\right], \\
L & =10^{-5} \times\left[\begin{array}{ll}
-0.1639 & 0.2791 \\
0.1262 & 0.0229
\end{array}\right] .
\end{aligned}
$$

Under the observer-based bounded controller, simulation results are obtained for the two-dimension uncertain discrete time-delay system with nonlinearity and disturbance, as shown in Figures 2-7. It is shown in Figures 2 and 3 that the stability of the closed-loop system can be obtained under the proposed bounded controller. Figure 4 shows that the state estimation errors of $\tilde{x}_{1}(k)$ and $\tilde{x}_{2}(k)$ are small, and the outputs of the state estimator can estimate the system state effectively. The simulation results shown in Figures 5 and 6 indicate that the outputs of the disturbance observer can effectively and quickly approximate the unknown external disturbance. Figure 7 shows that the control input is bounded under the designed controller. From these simulation results of the example, we can know that the state estimator and the disturbance observer can well approximate the system state and the disturbance, and the designed bounded control scheme based on the state estimator and the disturbance observer is valid for the discrete-time system subject to input saturation, parameter uncertainties, nonlinearity, disturbance, and state and input time delays.

4.2. The Case for Three-Dimension System. Considering the following discrete-time uncertain time-delay system with 


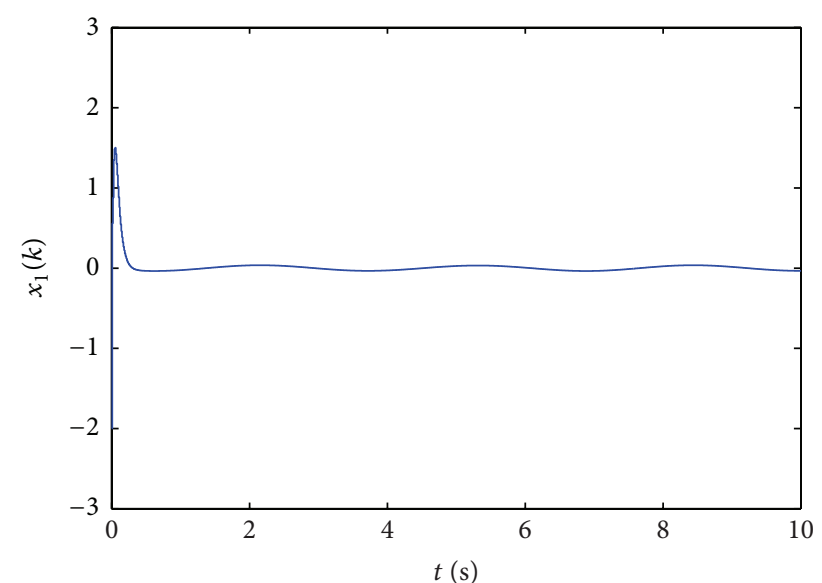

Figure 2: The system state $x_{1}(k)$.

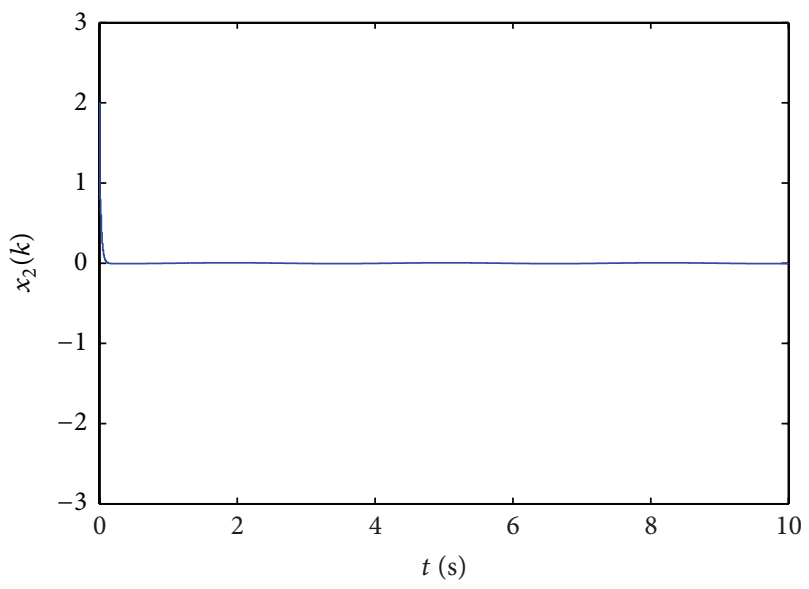

FIgURE 3: The system state $x_{2}(k)$.

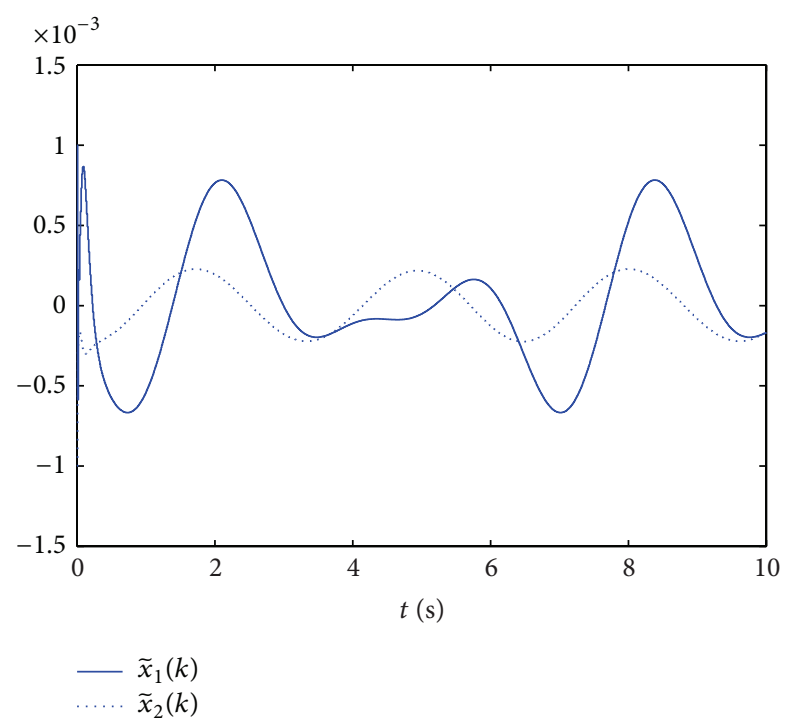

FiguRE 4: The estimation error outputs of $\tilde{x}_{1}(k)$ and $\tilde{x}_{2}(k)$.

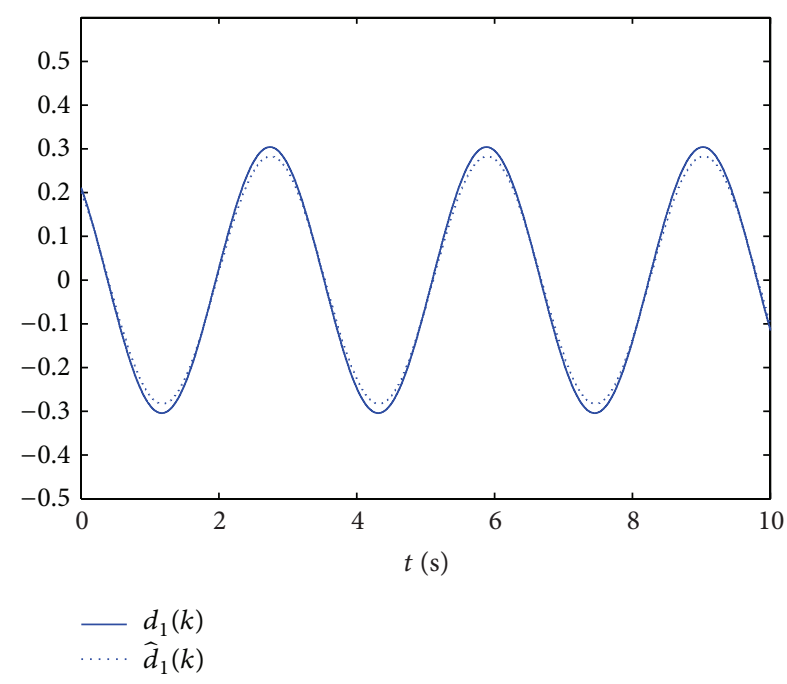

FIGURE 5: The disturbance $d_{1}(k)$ and the estimation output of $\widehat{d}_{1}(k)$.

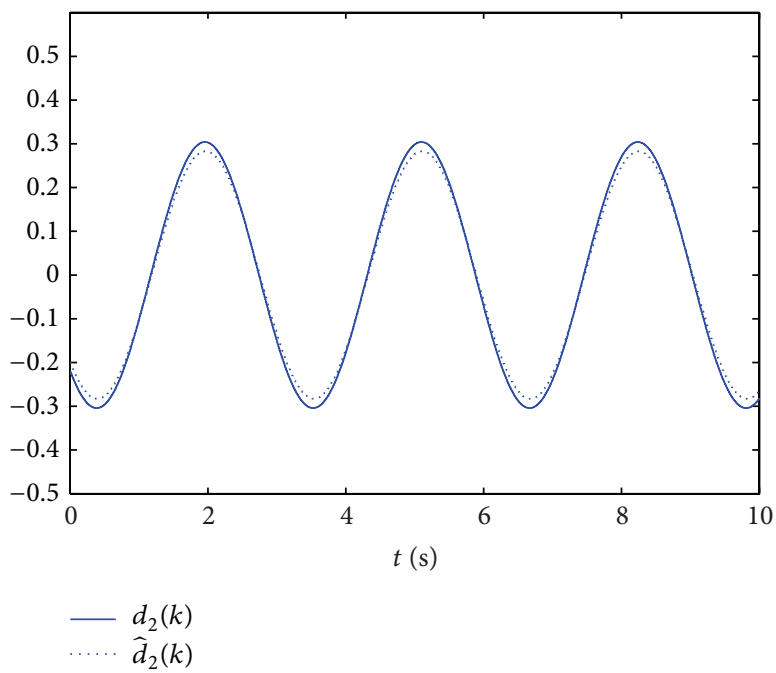

FIGURE 6: The disturbance $d_{2}(k)$ and the estimation output of $\widehat{d}_{2}(k)$.

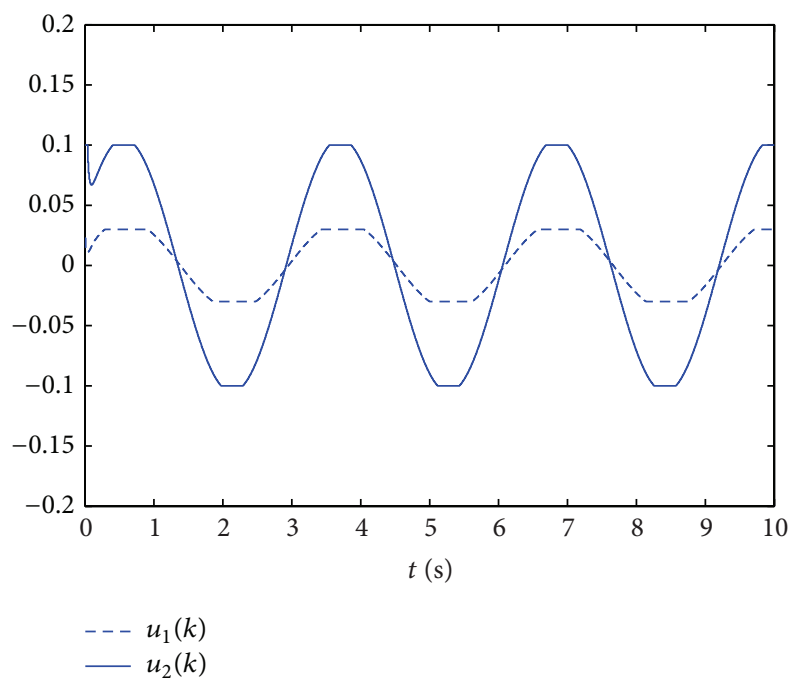

FIGURE 7: The control inputs $u_{1}(k)$ and $u_{2}(k)$. 
disturbance and nonlinearity, originally given by [41], the parameter matrices of system (1) are

$$
\begin{aligned}
& G_{0}=\left[\begin{array}{ccc}
0.7 & 0 & -0.5 \\
0.05 & 0.8 & 0 \\
0 & 0.3 & 0.6
\end{array}\right], \\
& G_{0 d}=\left[\begin{array}{ccc}
-0.02 & 0 & 0 \\
0 & -0.01 & 0.01 \\
0 & 0 & -0.02
\end{array}\right] \text {, } \\
& D=\left[\begin{array}{c}
0.1 \\
0 \\
0.2
\end{array}\right] \text {, } \\
& H_{0}=\left[\begin{array}{cc}
0.01 & 0 \\
0 & 0.01 \\
-0.012 & 0.12
\end{array}\right] \text {, } \\
& M_{1}=\left[\begin{array}{c}
0.2 \\
0 \\
0.3
\end{array}\right]^{T} \text {, } \\
& M_{2}=\left[\begin{array}{c}
0 \\
0.1 \\
0.01
\end{array}\right]^{T} \text {, } \\
& F_{01}=\left[\begin{array}{c}
0.1 \\
0 \\
0
\end{array}\right] \text {, } \\
& f_{01}(x(k), k)=\sin \left(2 x_{2}(k)\right) \text {. }
\end{aligned}
$$

The parameter matrices of exogenous system (5) are chosen as

$$
\begin{aligned}
& W=\left[\begin{array}{ccc}
0.9998 & 0.0200 & 0 \\
-0.0200 & 0.9998 & 0 \\
0 & 0 & 0.9802
\end{array}\right], \\
& V=\left[\begin{array}{lll}
1 & 0 & 0 \\
0 & 1 & 0
\end{array}\right] .
\end{aligned}
$$

Suppose that the initial values of state variable are $x(0)=\left[\begin{array}{lll}2 & -2 & 2.5\end{array}\right]^{T}$, the initial values of state estimator are $\widehat{x}(0)=\left[\begin{array}{lll}2.001 & -2.001 & 2.499\end{array}\right]^{T}$, the initial values of exogenous inputs are $w(0)=\left[\begin{array}{lll}0.11 & 0.22 & 0\end{array}\right]^{T}$, and $\widehat{w}(0)=$ $\left[\begin{array}{lll}0.1 & 0.21 & 0.01\end{array}\right]^{T}$. Choosing $U_{01}=\operatorname{diag}\left\{\begin{array}{lll}0 & 0.2 & 0\end{array}\right\}, T=1$,

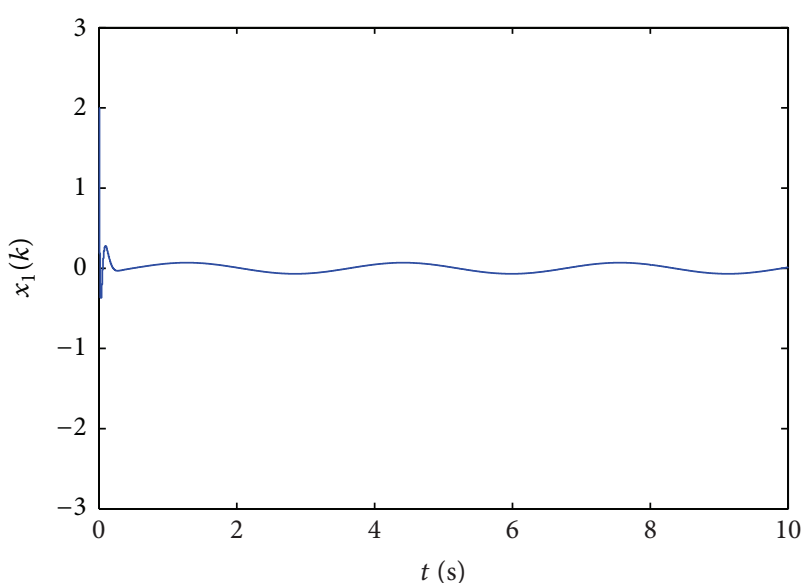

FIGURE 8: The system state $x_{1}(k)$.

$u_{1 \max }=0.01, u_{2 \max }=0.08, \lambda_{1}=0.1$, and $\lambda_{2}=\varepsilon=10$, by solving (23), we have

$$
\begin{aligned}
& K_{1}=\left[\begin{array}{lll}
-0.0029 & -0.0601 & 0.0482 \\
-0.0009 & -0.0191 & 0.0154
\end{array}\right] \text {, } \\
& K_{2}=\left[\begin{array}{cc}
-0.0087 & 0.0242 \\
0.0242 & -0.3696
\end{array}\right] \text {, } \\
& L_{1}=\left[\begin{array}{ccc}
0.6584 & -0.2042 & -0.3962 \\
-0.0430 & 0.7710 & 0.0020 \\
-0.9330 & 3.3798 & 0.3040
\end{array}\right] \text {, } \\
& L=10^{-3} \times\left[\begin{array}{ccc}
-0.0124 & -0.0111 & -0.0066 \\
0.0844 & -0.4582 & 0.1109 \\
-0.0000 & 0.0001 & -0.0000
\end{array}\right]
\end{aligned}
$$

Using the developed bounded controller, simulation results for the three-dimension uncertain discrete time-delay system with nonlinearity and disturbance are shown in Figures 8-14. From Figures 8-10, we can observe that the stability of the closed-loop system can be guaranteed under the observer-based bounded controller. According to Figure 11, we can see that the outputs of the state estimator can estimate the system state effectively. The disturbance estimate outputs $\widehat{d}_{1}(k), \widehat{d}_{2}(k)$ and the disturbance $d_{1}(k), d_{2}(k)$ are presented in Figures 12 and 13 which illustrate the effectiveness of the proposed disturbance observer. Figure 14 indicates that the control input is bounded under the designed controller. Based on these simulation results, we can obtain that the state estimator and the disturbance observer can effectively approximate the system state and the disturbance, and the proposed observer-based bounded control is valid for the discrete-time uncertain nonlinear system with time delays and disturbance. 


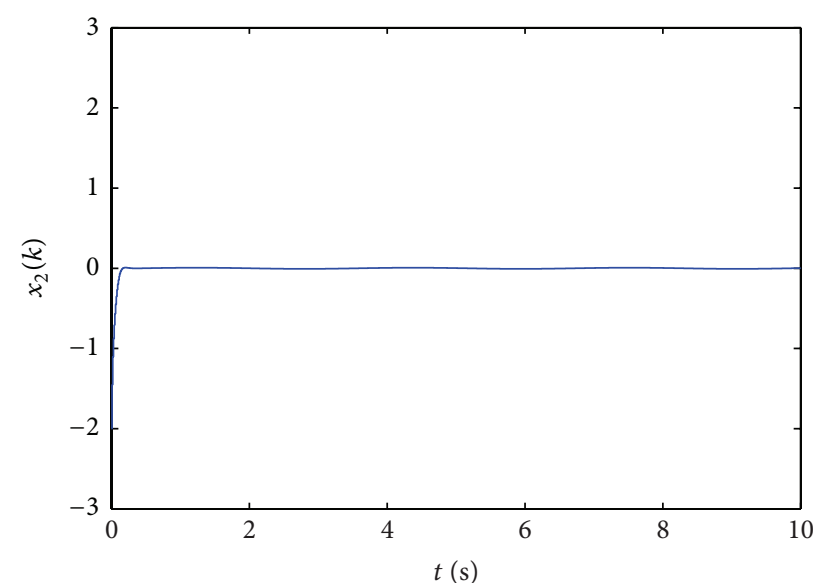

Figure 9: The system state $x_{2}(k)$.

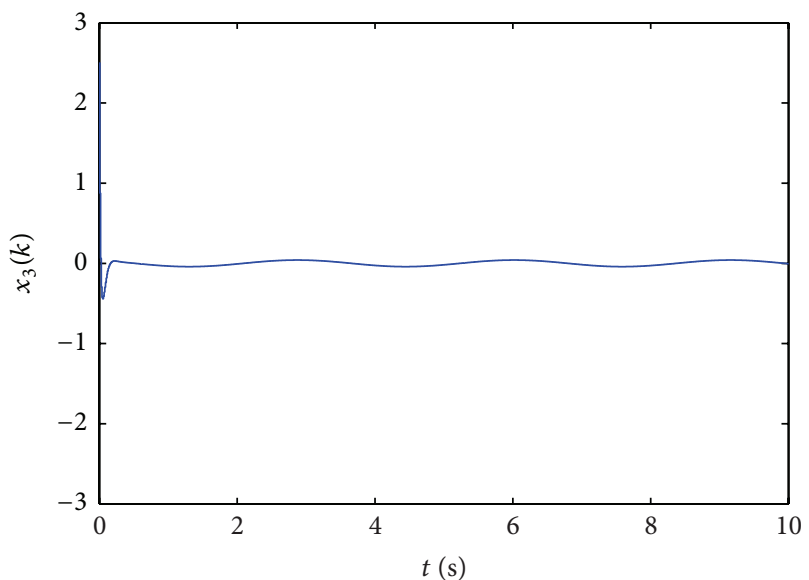

FIgURE 10: The system state $x_{3}(k)$.

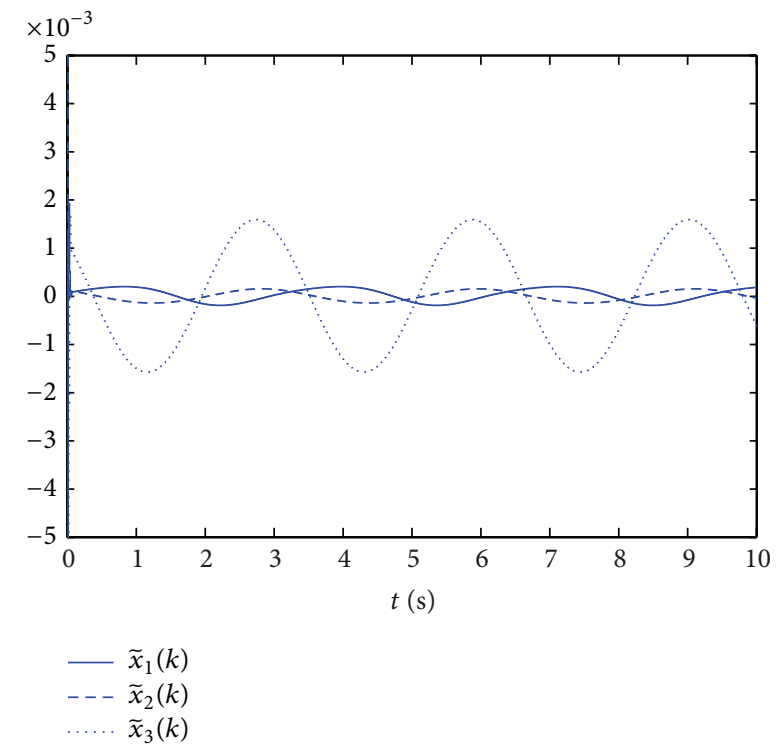

Figure 11: The estimation error outputs of $\tilde{x}_{1}(k), \tilde{x}_{2}(k)$, and $\tilde{x}_{3}(k)$.

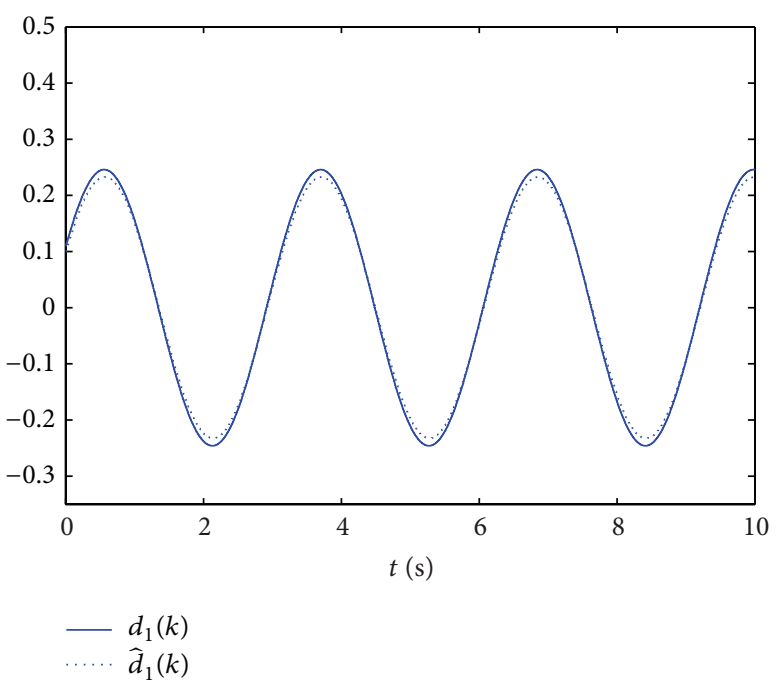

FIGURE 12: The disturbance $d_{1}(k)$ and the estimation output of $\widehat{d}_{1}(k)$.

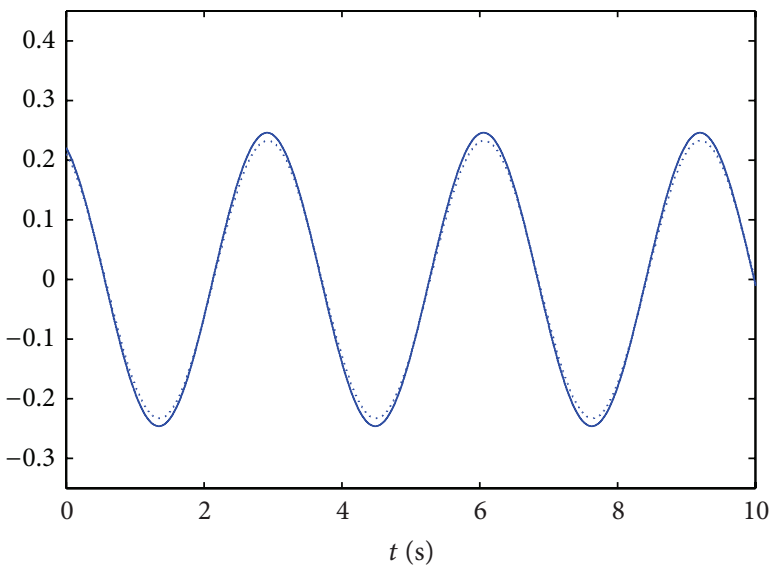

$$
-d_{2}(k)
$$

FIGURE 13: The disturbance $d_{2}(k)$ and the estimation output of $\widehat{d}_{2}(k)$.

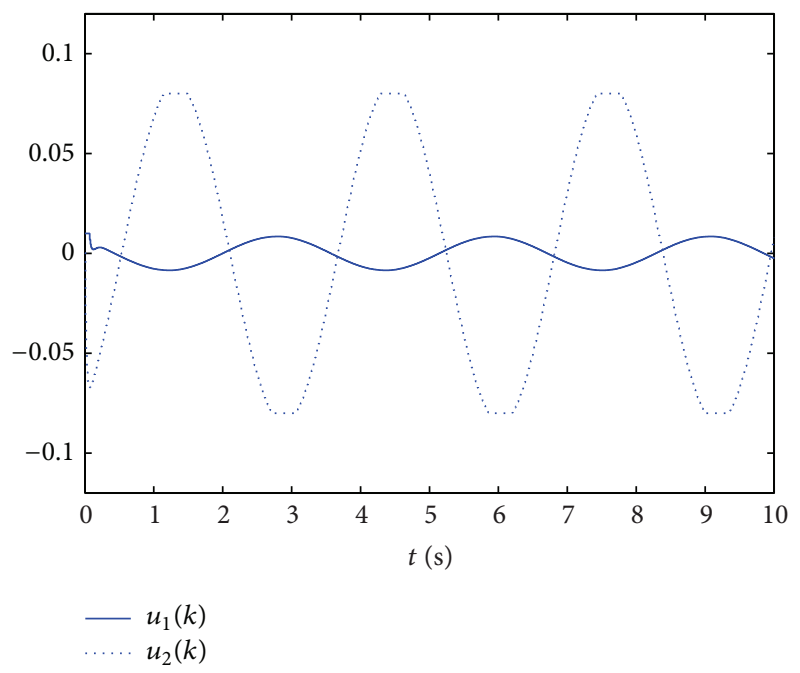

FIGURE 14: The control inputs $u_{1}(k)$ and $u_{2}(k)$. 


\section{Conclusion}

Stability of the uncertain discrete time-delay systems with disturbance and nonlinearity has been studied in this paper. The state estimator and the disturbance observer have been designed to estimate the unmeasured state vector and the unknown disturbance generated by the exogenous system. A bounded controller has been developed by the outputs of the state estimator and the disturbance observer. The stability of the closed-loop system has been proved via LMIs. Simulation examples have been provided to show the effectiveness of the proposed scheme. In the future, the time-varying state delay and input delay can be further considered for the discretetime uncertain nonlinear systems with disturbance and input saturation.

\section{Conflict of Interests}

The authors declare that there is no conflict of interests regarding the publication of this paper.

\section{Acknowledgments}

This research is supported by National Nature Science Foundation of China (no. 61573184), Jiangsu Natural Science Foundation of China (no. SBK20130033), Program for New Century Excellent Talents in University of China (no. NCET11-0830), and Specialized Research Fund for the Doctoral Program of Higher Education (no. 20133218110013).

\section{References}

[1] W.-H. Chen, Z.-H. Guan, and X. Lu, "Delay-dependent output feedback guaranteed cost control for uncertain time-delay systems," Automatica, vol. 40, no. 7, pp. 1263-1268, 2004.

[2] S.-P. Xiao, M. Wu, and J.-H. She, "Non-fragile delay-dependent $H_{\infty}$ control of linear time-delay system with uncertainties in state and control input," Journal of Central South University of Technology, vol. 15, no. 5, pp. 712-719, 2008.

[3] C. Yang, Z. Li, R. Cui, and B. Xu, "Neural network-based motion control of an underactuated wheeled inverted pendulum model," IEEE Transactions on Neural Networks and Learning Systems, vol. 25, no. 11, pp. 2004-2016, 2014.

[4] T. Senthilkumar and P. Balasubramaniam, "Delay-dependent robust $H_{\infty}$ control for uncertain stochastic T-S fuzzy systems with time-varying state and input delays," International Journal of Systems Science, vol. 42, no. 5, pp. 877-887, 2011.

[5] S. S. Ge, F. Hong, and T. H. Lee, "Adaptive neural network control of nonlinear systems with unknown time delays," IEEE Transactions on Automatic Control, vol. 48, no. 11, pp. 20042010, 2003.

[6] X. Li and J. Cao, "Adaptive synchronization for delayed neural networks with stochastic perturbation," Journal of the Franklin Institute. Engineering and Applied Mathematics, vol. 345, no. 7, pp. 779-791, 2008.

[7] X. Han, E. Fridman, and S. K. Spurgeon, "Sliding mode control in the presence of input delay: a singular perturbation approach," Automatica, vol. 48, no. 8, pp. 1904-1912, 2012.

[8] Y. Kao, J. Xie, L. Zhang, and H. Karimi, "A sliding mode approach to robust stabilisation of Markovian jump linear time-delay systems with generally incomplete transition rates," Nonlinear Analysis: Hybrid Systems, vol. 17, pp. 70-80, 2015.

[9] M. Chen and W.-H. Chen, "Disturbance-observer-based robust control for time delay uncertain systems," International Journal of Control, Automation and Systems, vol. 8, no. 2, pp. 445-453, 2010.

[10] M. Chen and J. Yu, "Disturbance observer-based adaptive sliding mode control for near-space vehicles," Nonlinear Dynamics, 2015.

[11] M. Chen and S. Ge, "Adaptive neural output feedback control of uncertain nonlinear systems with unknown hysteresis using disturbance observer," IEEE Transactions on Industrial Electronics, 2015.

[12] M. Chen, B. Ren, Q. Wu, and C. Jiang, "Anti-disturbance control of hypersonic flight vehicles with input saturation using disturbance observer," Science China Information Sciences, vol. 58, no. 7, pp. 1-12, 2015.

[13] M. Chen and J. Yu, "Adaptive dynamic surface control of NSVs with input saturation using a disturbance observer," Chinese Journal of Aeronautics, vol. 28, no. 3, pp. 853-864, 2015.

[14] Q. Yang and M. Chen, "Robust control for uncertain linear system subject to input saturation," Journal of Applied Mathematics, vol. 2014, Article ID 803842, 12 pages, 2014.

[15] J. Hu, Z. Wang, Y. Niu, and H. Gao, "Sliding mode control for uncertain discrete-time systems with Markovian jumping parameters and mixed delays," Journal of the Franklin Institute, vol. 351, no. 4, pp. 2185-2202, 2014.

[16] Y. He, G.-P. Liu, D. Rees, and M. Wu, " $H_{\infty}$ filtering for discretetime systems with time-varying delay," Signal Processing, vol. 89, no. 3, pp. 275-282, 2009.

[17] X. G. Liu, R. R. Martin, M. Wu, and M. L. Tang, "Delaydependent robust stabilisation of discrete-time systems with time-varying delay," IEE Proceedings: Control Theory and Applications, vol. 153, no. 6, pp. 689-702, 2006.

[18] P. Shi, Y. Zhang, and R. K. Agarwal, "Stochastic finite-time state estimation for discrete time-delay neural networks with Markovian jumps," Neurocomputing, vol. 151, no. 1, pp. 168-174, 2015.

[19] H. Li, C. Wang, P. Shi, and H. Gao, "New passivity results for uncertain discrete-time stochastic neural networks with mixed time delays," Neurocomputing, vol. 73, no. 16-18, pp. 3291-3299, 2010.

[20] S. Xu, J. Lu, S. Zhou, and C. Yang, "Design of observers for a class of discrete-time uncertain nonlinear systems with time delay," Journal of the Franklin Institute, vol. 341, no. 3, pp. 295-308, 2004.

[21] B. Xu, C. Yang, and Z. Shi, "Reinforcement learning output feedback NN control using deterministic learning technique," IEEE Transactions on Neural Networks and Learning Systems, vol. 25, no. 3, pp. 635-641, 2014.

[22] C. Yang, L. Zhai, S. G. Shuzhi, T. Chai, and H. L. Tong, "Adaptive model reference control of a class of MIMO discrete-time systems with compensation of nonparametric uncertainty," in Proceedings of the American Control Conference (ACC '08), pp. 4111-4116, Seattle, Wash, USA, June 2008.

[23] B. Xu, X. Huang, D. Wang, and F. Sun, "Dynamic surface control of constrained hypersonic flight models with parameter estimation and actuator compensation," Asian Journal of Control, vol. 16, no. 1, pp. 162-174, 2014.

[24] C. Yang, H. Ma, and M. Fu, "Adaptive predictive control of periodic non-linear auto-regressive moving average systems 
using nearest-neighbour compensation," IET Control Theory \& Applications, vol. 7, no. 7, pp. 936-951, 2013.

[25] S. Wo, Y. Zou, M. Sheng, and S. Xu, "Robust control for discretetime singular large-scale systems with parameter uncertainty," Journal of the Franklin Institute, vol. 344, no. 2, pp. 97-106, 2007.

[26] B. Xu, C. Yang, and Y. Pan, "Global neural dynamic surface tracking control of strict-feedback systems with application to hypersonic flight vehicle," IEEE Transactions on Neural Networks and Learning Systems, vol. 26, no. 10, pp. 2563-2575, 2015.

[27] C. Yang, S. S. Ge, C. Xiang, T. Chai, and T. H. Lee, "Output feedback NN control for two classes of discrete-time systems with unknown control directions in a unified approach," IEEE Transactions on Neural Networks, vol. 19, no. 11, pp. 1873-1886, 2008.

[28] C. Yang, S. S. Ge, and T. H. Lee, "Output feedback adaptive control of a class of nonlinear discrete-time systems with unknown control directions," Automatica, vol. 45, no. 1, pp. 270276, 2009.

[29] Y. Xia, Z. Zhu, C. Li, H. Yang, and Q. Zhu, "Robust adaptive sliding mode control for uncertain discrete-time systems with time delay," Journal of the Franklin Institute, vol. 347, no. 1, pp. 339-357, 2010.

[30] S. Li, J. Yang, W. Chen, and X. Chen, Disturbance ObserverBased Control: Methods and Applications, CRC Press, Boca Raton, Fla, USA, 2014.

[31] K. Ohishi, M. Nakao, K. Ohnishi, and K. Miyachi, "Microprocessor-controlled DC motor for load-insensitive position servo system," IEEE Transactions on Industrial Electronics, vol. 34, no. 1, pp. 44-49, 1987.

[32] A. Mohammadi, M. Tavakoli, H. J. Marquez, and F. Hashemzadeh, "Nonlinear disturbance observer design for robotic manipulators," Control Engineering Practice, vol. 21, no. 3, pp. 253-267, 2013.

[33] K.-S. Kim and K.-H. Rew, "Reduced order disturbance observer for discrete-time linear systems," Automatica, vol. 49, no. 4, pp. 968-975, 2013.

[34] H. Sun and L. Hou, "Composite anti-disturbance control for a discrete-time time-varying delay system with actuator failures based on a switching method and a disturbance observer," Nonlinear Analysis. Hybrid Systems, vol. 14, pp. 126-138, 2014.

[35] X. Wei, H. Zhang, and L. Guo, "Saturating composite disturbance-observer-based control and $H_{\infty}$ control for discrete time-delay systems with nonlinearity," International Journal of Control, Automation and Systems, vol. 7, no. 5, pp. 691-701, 2009.

[36] B. Xu, Z. Shi, and C. Yang, "Composite fuzzy control of a class of uncertain nonlinear systems with disturbance observer," Nonlinear Dynamics, vol. 80, no. 1-2, pp. 341-351, 2015.

[37] W. Wang and H.-S. Zhang, " $H_{\infty}$ control for discrete-time system with input time-varying delay," Acta Automatica Sinica, vol. 36, no. 4, pp. 597-602, 2010.

[38] A. Gonzalez, A. Sala, and P. Albertos, "Predictor-based stabilization of discrete time-varying input-delay systems," Automatica, vol. 48, no. 2, pp. 454-457, 2012.

[39] A. González, "Robust stabilization of linear discrete-time systems with time-varying input delay," Automatica, vol. 49, no. 9, pp. 2919-2922, 2013.

[40] W.-H. Chen, Z.-H. Guan, and X. Lu, "Delay-dependent guaranteed cost control for uncertain discrete-time systems with both state and input delays," Journal of the Franklin Institute.
Engineering and Applied Mathematics, vol. 341, no. 5, pp. 419430, 2004.

[41] L. Yu and F. Gao, "Optimal guaranteed cost control of discretetime uncertain systems with both state and input delays," Journal of the Franklin Institute. Engineering and Applied Mathematics, vol. 338, no. 1, pp. 101-110, 2001.

[42] H. Zhang, L. Xie, and G. Duan, " $H_{\infty}$ control of discretetime systems with multiple input delays," IEEE Transactions on Automatic Control, vol. 52, no. 2, pp. 271-283, 2007.

[43] J. H. Kim, "Delay-dependent robust $H_{\infty}$ control for discretetime uncertain singular systems with interval time-varying delays in state and control input," Journal of the Franklin Institute. Engineering and Applied Mathematics, vol. 347, no. 9, pp. 1704-1722, 2010.

[44] V. Macián, J. M. Luján, C. Guardiola, and P. Yuste, "DFTbased controller for fuel injection unevenness correction in turbocharged diesel engines," IEEE Transactions on Control Systems Technology, vol. 14, no. 5, pp. 819-827, 2006.

[45] S. S. Ge, C. Yang, and T. H. Lee, "Adaptive predictive control using neural network for a class of pure-feedback systems in discrete time," IEEE Transactions on Neural Networks, vol. 19, no. 9, pp. 1599-1614, 2008.

[46] J. Solsona, M. I. Valla, and C. Muravchik, "A nonlinear reduced order observer for permanent magnet synchronous motors," IEEE Transactions on Industrial Electronics, vol. 43, no. 4, pp. 492-497, 1996.

[47] Y. Zhang, P. Shi, and S. K. Nguang, "Observer-based finite-time $H_{\infty}$ control for discrete singular stochastic systems," Applied Mathematics Letters, vol. 38, pp. 115-121, 2014.

[48] C. Yang, Y. Li, S. S. Ge, and T. Lee, "Adaptive control of a class of discretetime MIMO nonlinear systems with uncertain couplings," International Journal of Control, vol. 83, no. 10, pp. 2120-2133, 2010.

[49] A. Arunkumar, R. Sakthivel, K. Mathiyalagan, and S. M. Anthoni, "Robust state estimation for discrete-time BAM neural networks with time-varying delay," Neurocomputing, vol. 131, pp. 171-178, 2014.

[50] M. Boutayeb and M. Darouach, "A reduced-order observer for non-linear discrete-time systems," Systems \& Control Letters, vol. 39, no. 2, pp. 141-151, 2000.

[51] H.-N. Wu, "Delay-dependent $H_{\infty}$ fuzzy observer-based control for discretetime nonlinear systems with state delay," Fuzzy Sets and Systems. An International Journal in Information Science and Engineering, vol. 159, no. 20, pp. 2696-2712, 2008.

[52] Y. Zhang, P. Shi, S. K. Nguang, and H. R. Karimi, "Observerbased finite-time fuzzy $H_{\infty}$ control for discrete-time systems with stochastic jumps and time-delays," Signal Processing, vol. 97, pp. 252-261, 2014.

[53] X. Li and Z. Xiang, "Observer design of discrete-time impulsive switched nonlinear systems with time-varying delays," Applied Mathematics and Computation, vol. 229, pp. 327-339, 2014.

[54] S. Wang, Y. Jiang, Y. Li, and D. Liu, "Reliable observer-based $H_{\infty}$ control for discrete-time fuzzy systems with time-varying delays and stochastic actuator faults via scaled small gain theorem," Neurocomputing, vol. 147, pp. 251-259, 2015.

[55] L. Guo and W.-H. Chen, "Disturbance attenuation and rejection for systems with nonlinearity via DOBC approach," International Journal of Robust and Nonlinear Control, vol. 15, no. 3, pp. 109-125, 2005.

[56] G. Tang, H. Sun, and H. Pang, "Approximately optimal tracking control for discrete time-delay systems with disturbances," Progress in Natural Science, vol. 18, no. 2, pp. 225-231, 2008. 
[57] P. Shi, E.-K. Boukas, Y. Shi, and R. K. Agarwal, "Optimal guaranteed cost control of uncertain discrete time-delay systems," Journal of Computational and Applied Mathematics, vol. 157, no. 2, pp. 435-451, 2003.

[58] L. Zhu, Y. Shen, and C. Li, "Finite-time control of discrete-time systems with time-varying exogenous disturbance," Communications in Nonlinear Science and Numerical Simulation, vol. 14, no. 2, pp. 361-370, 2009.

[59] G. Song and Z. Wang, "A delay partitioning approach to output feedback control for uncertain discrete time-delay systems with actuator saturation," Nonlinear Dynamics, vol. 74, no. 1, pp. 189202, 2013.

[60] Q. Zheng and F. Wu, "Output feedback control of saturated discrete-time linear systems using parameter-dependent Lyapunov functions," Systems \& Control Letters, vol. 57, no. 11, pp. 896-903, 2008. 


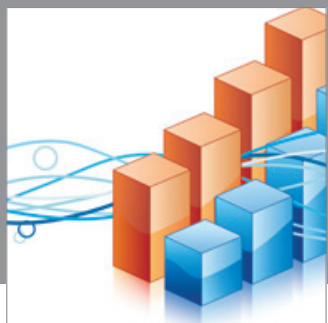

Advances in

Operations Research

mansans

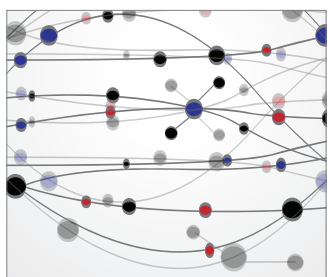

The Scientific World Journal
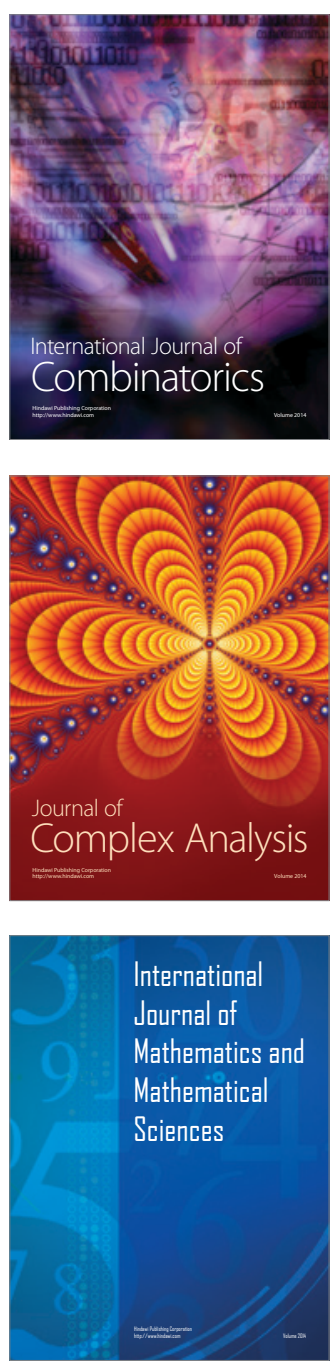
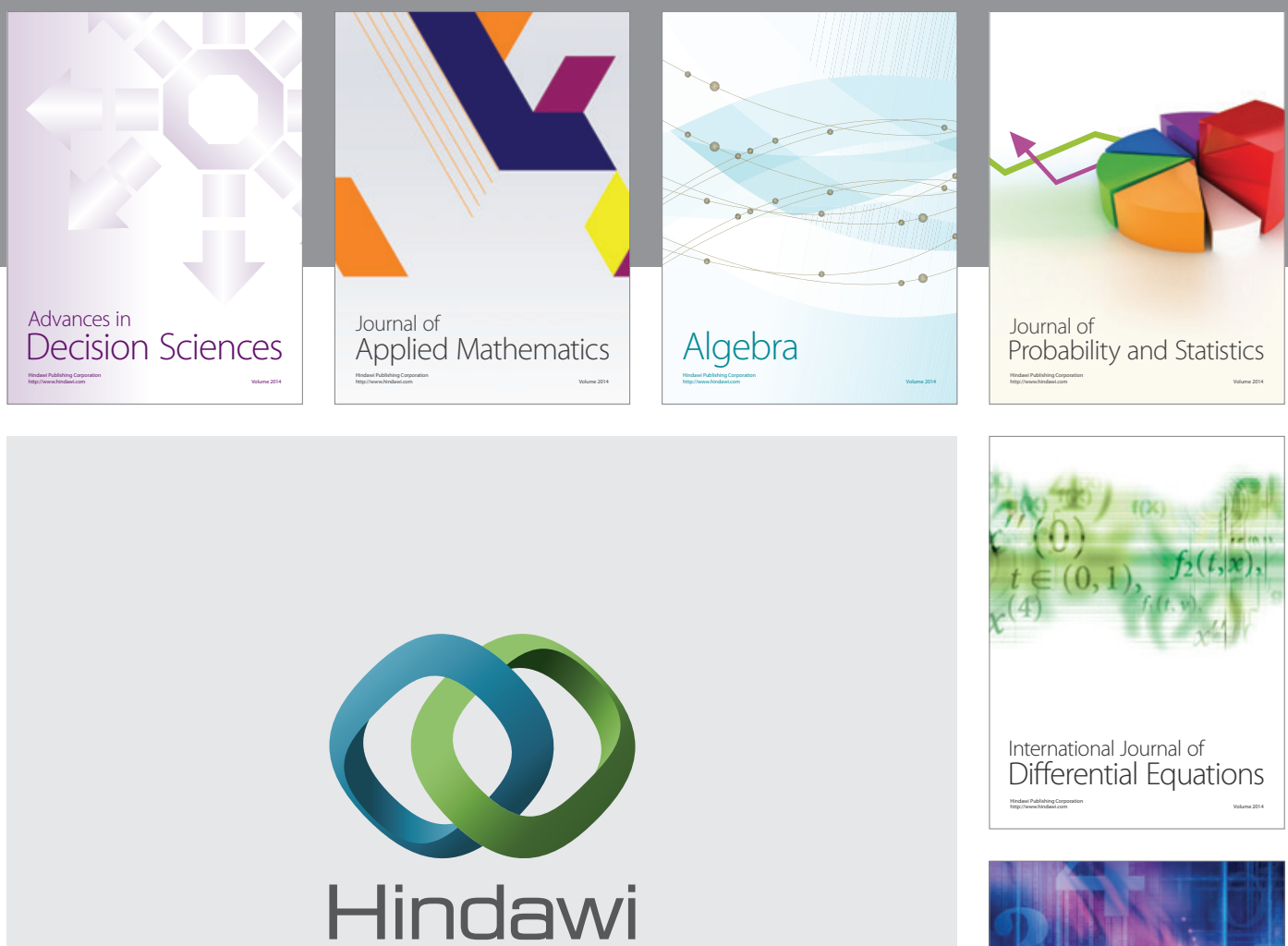

Submit your manuscripts at http://www.hindawi.com
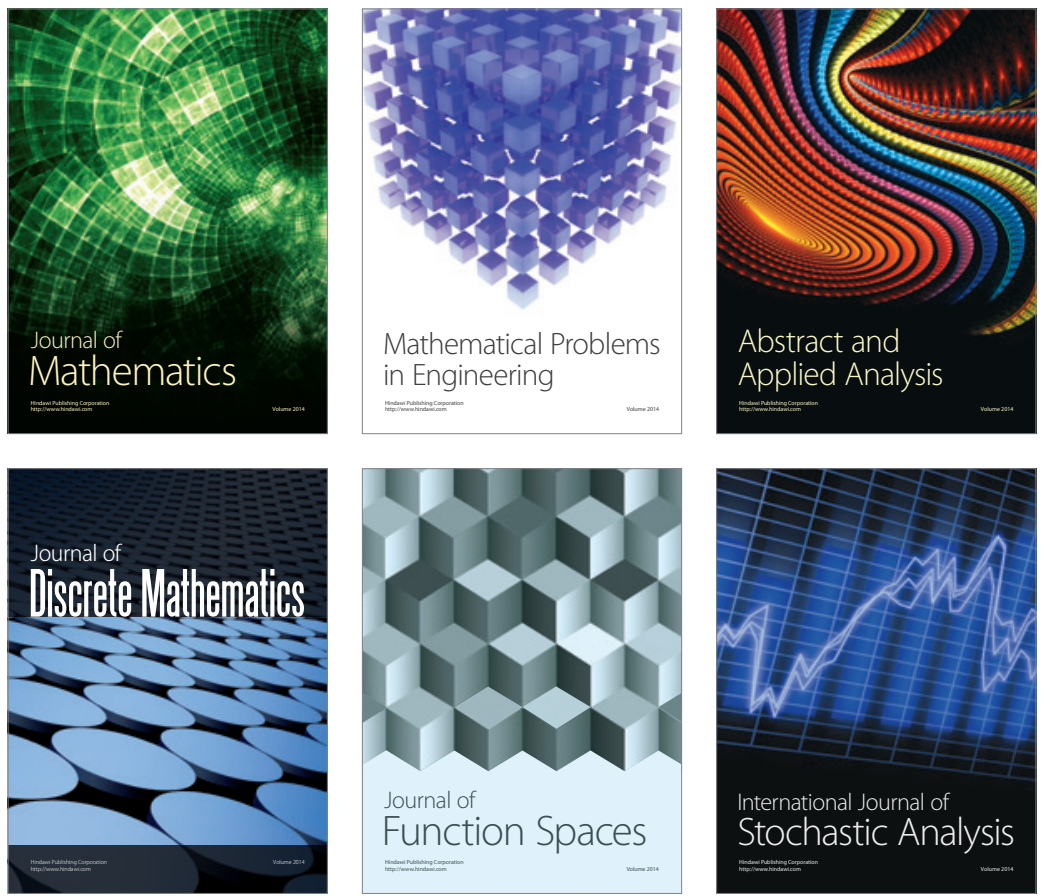

Journal of

Function Spaces

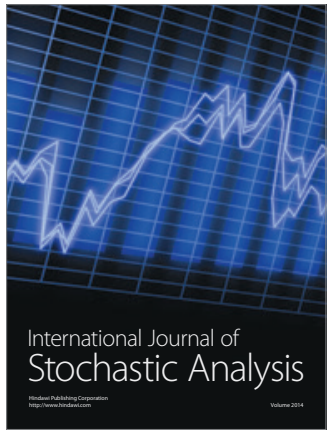

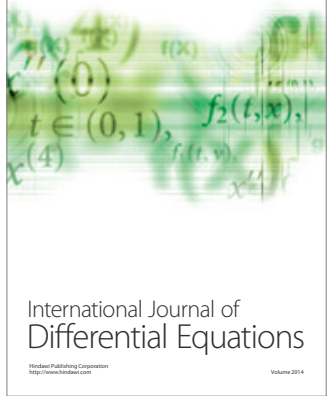
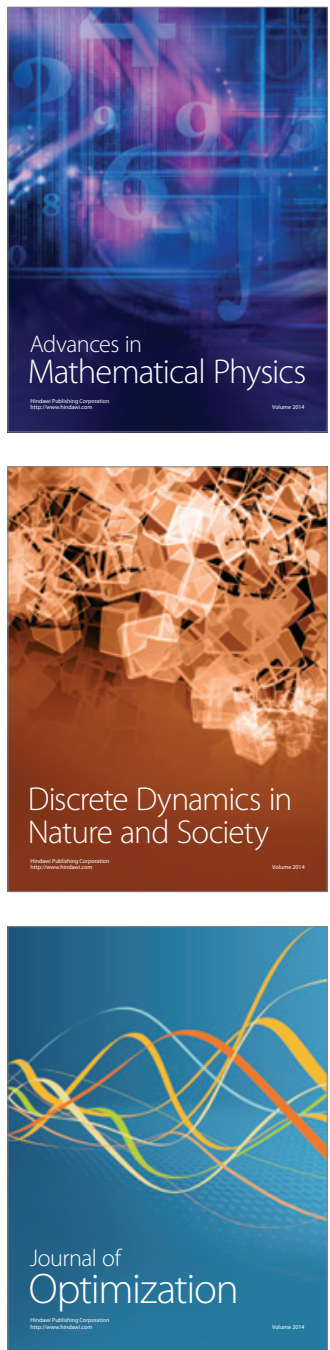